\title{
The Association Between Body Mass Index and Glycemic Control in Patients with Type 2 Diabetes Across Eight Countries: A Literature Review
}

\author{
Tessa Kennedy-Martin ${ }^{1}$, Kristina S Boye ${ }^{2 *}$ and Matthew Kennedy-Martin ${ }^{1}$ \\ ${ }^{1}$ Kennedy Martin Health Outcomes (KMHO) Limited, UK \\ ${ }^{2}$ Eli Lilly, USA
}

Submission: July 29, 2021; Published: August 10, 2021

*Corresponding author: Kristina S Boye, Global Patient Outcomes and Real-World Evidence, Eli Lilly, 1 Lilly Corporate Ctr, Indianapolis, IN 46285, USA. Email: boye_kristina_secnik@lilly.com

\section{Abstract}

Objective: To investigate the association between obesity and glycemic control based on country-specific glycemic control data from the USA and selected European and Asian countries by body mass index (BMI), or BMI data by glycemic control $\left(\mathrm{HbA}_{1 \mathrm{c}}\right.$ ), in individuals with type 2 diabetes (T2D).

Methods: Literature search of databases and abstracts to identify real-world studies (2015-2020) reporting $\mathrm{HbA}_{1 \mathrm{c}}$ and BMI data.

Results: Seventeen articles (China, including Hong Kong, $n=10$; Japan, $n=3$; USA, $n=2$; Spain, $n=1 ;$ UK, $n=1$ ) were identified from 6696 deduplicated references. Of the 13 studies reporting by BMI mean rates of glycemic control or likelihood of achieving glycemic control, eight found that achieving control significantly declined as BMI increased; one reported a clear trend but no statistics; two reported glycemic control rates for those with obesity to be significantly worse than for one other group; and two reported no significant difference with BMI. Of the six studies reporting average $\mathrm{HbA}_{1 \mathrm{c}}$ levels by $\mathrm{BMI}$, four reported $\mathrm{HbA}_{1 \mathrm{c}}$ to increase with BMI. Two of three studies reporting mean BMIs by glycemic control found these increased with $\mathrm{HbA}_{1 c}$.

Conclusions: This review consolidates recent evidence on the glycemic control status of individuals with T2D reported by BMI in selected countries. Studies generally reported a lower chance of achieving glycemic control targets in those with overweight or obesity. Most studies found greater $\mathrm{HbA}_{1 \mathrm{c}}$ in individuals with higher $\mathrm{BMI}$, and average BMI greater in those with higher levels of $\mathrm{HbA}_{1 \mathrm{c}}$. Focused efforts are needed to improve glycemic control in patients with T2D and overweight/obesity.

Keywords: Body mass index; China; Europe; Glycemic control; Japan; Type 2 diabetes; United States; Weight

Abbreviations: ADA: American Diabetes Association; BMI: Body Mass Index; CVD: Cardiovascular disease; EMR: Electronic Medical Record; FPG: Fasting Plasma Glucose; $\mathrm{HbA}_{1 \mathrm{c}}$ : Glycated Hemoglobin; ISPOR: Professional Society for Health Economics and Outcomes Research; NA: Not Applicable; OR: Odds Ratio; P: Percentile; RR: Relative Risk; RWE: Real-World Evidence; T1D: Type 1 Diabetes; T2D: Type 2 Diabetes; WHO: World Health Organization

\section{Introduction}

Type 2 diabetes (T2D) and obesity are intrinsically linked. Obesity is a major risk factor for developing T2D, and the progression of disease increases with obesity [1]. Obesity is also known to increase the odds of developing many common complications of diabetes, including heart disease, retinopathy, dyslipidemia, and hypertension [2].

Hyperglycemia is the defining feature of T2D, so achieving glycemic control is a fundamental aim of disease management. Inadequate control of $\mathrm{T} 2 \mathrm{D}$ can result in the development of disabling and life-threatening complications [3,4]. However, a large proportion of individuals are still failing to achieve glycemic control and a significant proportion of those who are not achieving glycemic control have overweight or obesity [5]. Given the close relationship between T2D and obesity, weight reduction is a key therapeutic goal in both the prevention and management of type 2 diabetes $[1,6]$, with weight management playing a prominent role in T2D guidelines. Such guidelines outline that all individuals with T2D and overweight or obesity should be advised of the health benefits of weight loss and be encouraged to engage in a program 


\section{Current Research in Diabetes \& Obesity Journal}

of intensive lifestyle management to include dietary restrictions and increases in physical activity [4,7]. They also emphasize that when choosing a glucose-lowering agent for patients with overweight or obesity, careful consideration should be given to its impact on weight $[4,7]$.

Weight loss is recognized to be associated with improvements in glycemic control among individuals with T2D [8]. However, weight reduction or maintaining a healthy weight can be challenging for many people with T2D. For example, in the United States it has been estimated that $89.0 \%$ of adults with diabetes had overweight or obesity [9].

With high rates of overweight and obesity in individuals with
T2D, and the ongoing development of new diabetes therapies, having data from recent real-world studies on body mass index (BMI) and glycemic control would improve understanding of the patient population and could help to inform future decisionmaking. Therefore, the purpose of this review was to identify and report recent studies on the association between BMI and glycemic control through the identification of recently published country-specific (China, France, Germany, Italy, Japan, Spain, UK, USA) studies. The review is intended to serve as a resource of available studies for those working in or with those countries. There is no attempt to aggregate or pool data across the different studies, given the differences in study populations and also BMI cut-offs that are used in different countries.

\section{Methods}

Table 1: Eligibility criteria for the review.

\begin{tabular}{|c|c|c|}
\hline Study Characteristic & Eligible & Ineligible \\
\hline Patient population & $\begin{array}{l}\text { Adult ( } \geq 18 \text { years) patients with T2D Study undertaken in } \\
\text { China, } \\
\text { France, Germany, Italy, Japan, Spain, UK, or USA }\end{array}$ & $\begin{array}{l}\text { Pediatric }(<18 \text { years) patients } \\
\text { Study undertaken in a country other than China, France, } \\
\text { Germany, Italy, Japan, Spain, UK, or USA }\end{array}$ \\
\hline \multirow{3}{*}{ Outcomes } & $\mathrm{HbA}_{1 \mathrm{c}}$ & \multirow{3}{*}{ Outcomes unrelated to $\mathrm{HbA}_{1 \mathrm{c}}$ or $\mathrm{BMI} /$ weight } \\
\hline & BMI & \\
\hline & Weight & \\
\hline \multirow{7}{*}{ Study type } & \multirow{7}{*}{$\begin{array}{c}\text { Real-world cross-sectional study } \\
\text { Real-world case-control study } \\
\text { Real-world cohort study } \\
\text { Administrative or claims database study } \\
\text { Real-world electronic health record (EHR) } \\
\text { Registry study representing real-world clinical practice } \\
\text { Questionnaires and surveys relating to real-world clinical } \\
\text { practice }\end{array}$} & $\begin{array}{l}\text { Non-observational studies that do not reflect real-world } \\
\text { clinical practice }\end{array}$ \\
\hline & & Case studies \\
\hline & & Pragmatic or randomized controlled trials \\
\hline & & Utility studies \\
\hline & & $\begin{array}{l}\text { Preference or satisfaction studies based on hypothetical } \\
\text { profiles }\end{array}$ \\
\hline & & Reviews \\
\hline & & $\begin{array}{l}\text { Editorials/comments } \\
\text { Economic evaluations }\end{array}$ \\
\hline Time frame & Start January 2015 to end December 2019 & Outside of included date range \\
\hline Language & English (abstract) & Non-English abstract \\
\hline
\end{tabular}

BMI: Body Mass Index; $\mathrm{HbA}_{1 \mathrm{c}}$ : Glycated Hemoglobin; T2D: Type 2 Diabetes.

A literature review was conducted to identify studies reporting the relationship between BMI and glycemic control in adults with T2D. A robust and reproducible protocol was developed for the review that outlined the focus with respect to scope, patient population, appropriate study type, and outcomes of interest, and also provided details of the search strategy and data extraction methods. The protocol was developed to reduce the risk of introducing bias and for transparency.

\section{Search strategy}

Searches were undertaken for literature published in the English language from January 1, 2015, through January 8, 2020. The MEDLINE and EMBASE bibliographic databases were searched via OVID. A hand-search of the bibliographies of eligible publications was also undertaken to identify any relevant studies that, for whatever reason, were not found by the original search.
EMBASE includes congress abstracts and all those indexed from 2019 until the search date were included to identify studies that may not have reached full publication. The main search strategy consisted of three concepts: T2D AND real-world evidence (RWE) and [(glycemic control OR BMI OR obesity levels/weight categories) AND countries]. These were captured using subject headings and text word searches in title, abstract, and keyword heading word fields. Search terms for the T2D concept included terms for non-specific diabetes and terms for explicit T2D. In the context of this search, 'RWE' was evidence based on real-world data derived from the following types of study: retrospective, cross-sectional, or prospective. Included were case-control studies, cohort studies, administrative or claims database studies, electronic medical record (EMR) studies, registry studies, questionnaires, and surveys relating to real-world clinical practice. 


\section{Current Research in Diabetes \& Obesity Journal}

\section{Study eligibility criteria}

Eligibility criteria were prespecified and are described in Table 1.

\section{Study selection and data extraction}

Titles and abstracts of the search results were assessed for relevance to the research questions by two independent reviewers. Studies considered as meeting or possibly meeting the eligibility criteria were selected for further review using the fulltext record. Any disagreements between reviewers were resolved by discussion until consensus was reached. Data extraction was performed on a standardized data extraction form by one reviewer, with the second undertaking a quality review.

\section{Compliance with ethics guidelines}

This article is a review of previously published studies and does not include any new research on human or animal subjects performed by any of the authors.

\section{Results}

\section{Overview of search results}

A PRISMA diagram of the study selection process is presented in Figure 1. Database searches yielded 6696 de-duplicated records and after initial abstract and full-text review 17 articles remained for inclusion in the final dataset.

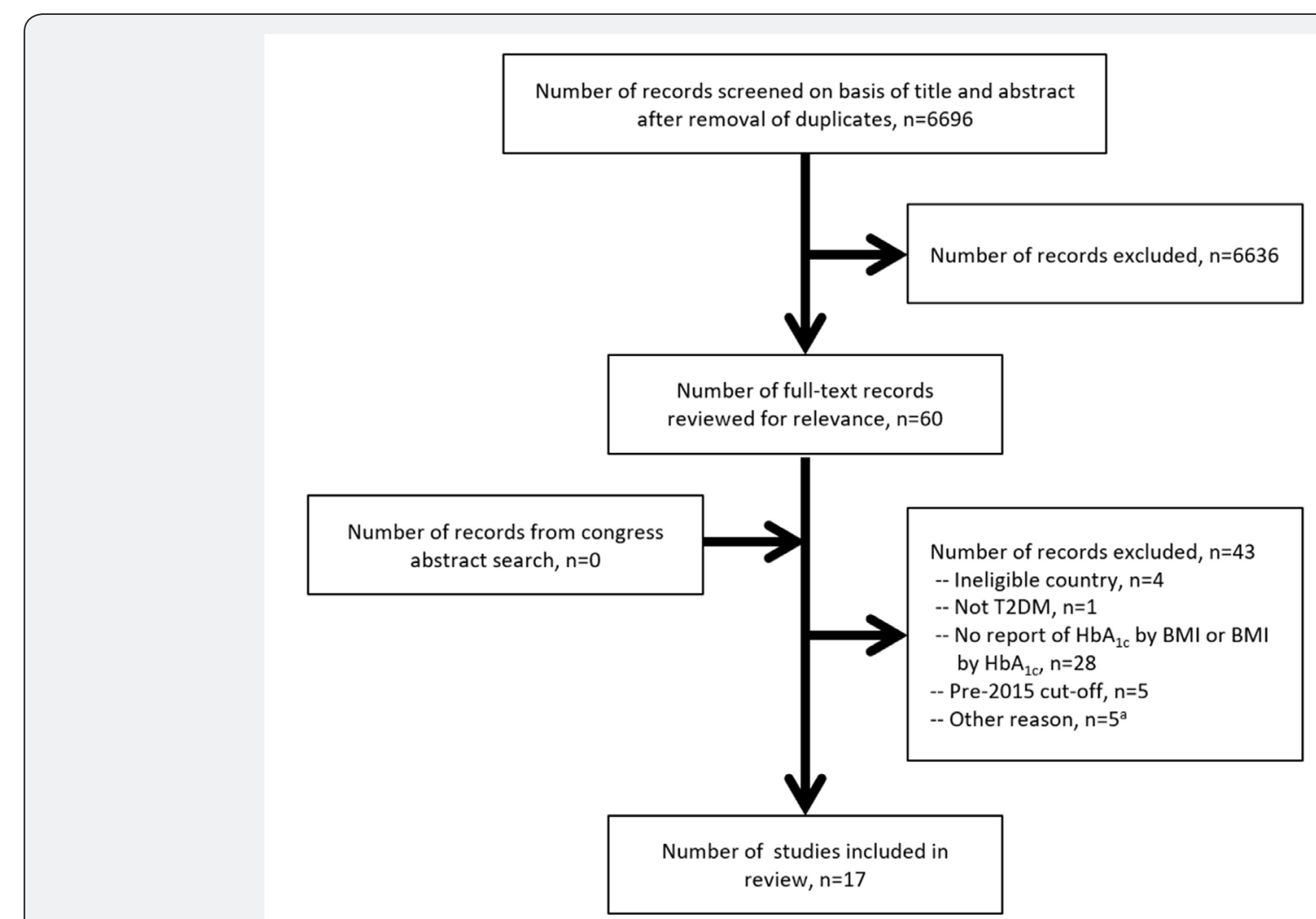

Figure 1: Study selection process.

${ }^{a}$ Other reasons ( $n=1$ record each) included: evaluation of progression between glycemic stages across different levels of BMl; evaluation of weight loss only (no BMI or $\mathrm{HbA}_{1 \mathrm{c}}$ ); population included individuals with $\mathrm{T} 2 \mathrm{D}$ and acute coronary syndrome; individuals included all types of diabetes; studied association between onset of diabetic kidney disease, $\mathrm{HbA}_{1 \mathrm{c}}$ and $\mathrm{BMI}$; evaluated glycemic level by percentage weight change rather than BMI

BMI: Body Mass Index; $\mathrm{HbA}_{1 \mathrm{c}}$ : Glycated Hemoglobin; T2D: Type 2 Diabetes

\section{Study characteristics}

There were 10 prospective studies and seven retrospective studies. Of the 10 prospective studies, six were cross-sectional and only gathered data at one time point [10-15]; four were longitudinal, but only baseline demographic data were used in our analysis [16-19]. Seven retrospective studies were identified [2,20-25]; in six of these, baseline data from the cohorts were used for our analysis; the other study [20] was different in that it looked at data over time, presenting the relative risk for having $\mathrm{HbA}_{1 \mathrm{c}}>7 \%$ presented by BMI category at 1-, 2-, 3-, and 5-year follow-up. Characteristics of included studies are summarized in
Table 2.

A range of different sources were used to capture the data relevant to our analysis. These included medical records [2,12,20$25]$, study data [10,14-19], and data from the Diabetes Specific Programme [11]. Most studies were from were from Asia (China, $n=9$; Japan, $n=3$; Hong Kong, $n=1)$, followed by the USA $(n=2)$, Spain $(n=1)$, and the UK $(n=1)$. No studies were identified in the other included countries. Most of the studies reported no sponsorship $(\mathrm{n}=12)$; some reported pharmaceutical company sponsorship $(n=5)$. 


\section{Current Research in Diabetes \& Obesity Journal}

The ways in which the data were reported varied (Table 2). The most common approach was to report glycemic control rates by BMI category ( $\mathrm{n}=9$ studies); some studies presented a regression analysis on higher BMI as a risk factor for worse glycemic control $(n=8)$. There was some overlap, with 13 studies reporting either or both. Three of the studies that presented a regression analysis also reported categorical BMI distribution for $\mathrm{HbA}_{1 \mathrm{c}}$ categories (two studies for controlled vs uncontrolled; one study for $\mathrm{HbA}_{1 \mathrm{c}}$ ranges). Mean $\mathrm{HbA}_{1 \mathrm{c}}$ was reported by BMI category ( $\mathrm{n}=6$ studies). Three studies reported average BMI by glycemic control.

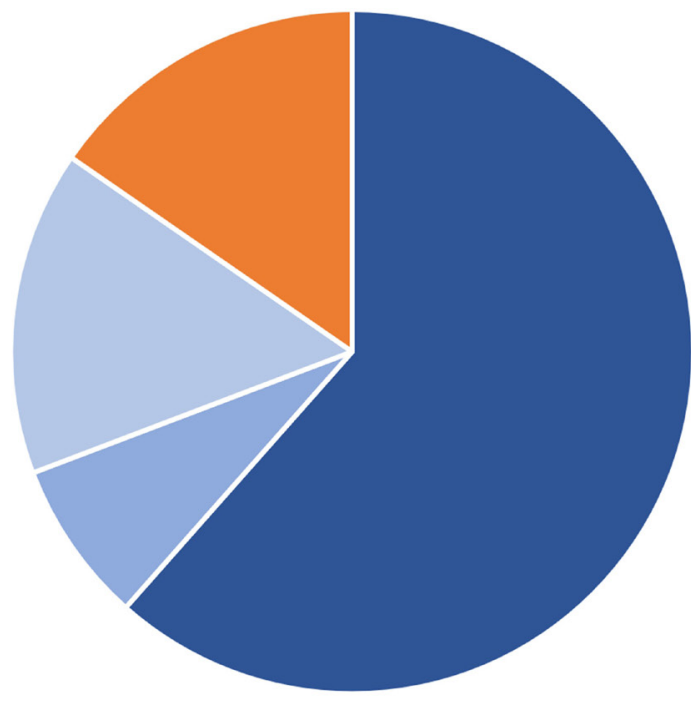

- Higher levels of BMI were significantly associated with worse glycemic control or higher BMI increased the likelihood of not achieving glycemic control $(n=8)$

- Trend shown that higher levels of BMI were associated with worse glycemic control $(n=1)$

- Some evidence that higher levels of BMI were associated with significantly worse glycemic control $(n=2)$

- Did not show that higher levels of BMI were associated with worse glycemic control $(n=2)$

Figure 2: Studies reporting glycemic control rates by BMI, and BMI as a factor in regression analyses for poor glycemic control.

Table 2: Overview of study methods and data reporting (split by country).

\begin{tabular}{|c|c|c|c|c|c|c|c|c|c|}
\hline \multirow{2}{*}{ Reference } & \multirow{2}{*}{$\begin{array}{l}\text { Study Type } \\
\text { (Sponsor) }\end{array}$} & \multirow{2}{*}{$\begin{array}{l}\text { Patient } \\
\text { Population } \\
\text { (Numbers) }\end{array}$} & \multirow{2}{*}{$\begin{array}{l}\text { Treatment } \\
\text { Profile of } \\
\text { Included } \\
\text { Patients }\end{array}$} & \multirow{2}{*}{$\begin{array}{c}\text { Source of } \\
\text { Data for } \\
\text { Weight/ } \\
\text { HbA }_{1 c} \\
\text { Analysis }\end{array}$} & \multicolumn{2}{|c|}{$\begin{array}{c}\text { Reporting by BMI } \\
\text { Category }\end{array}$} & \multirow[t]{2}{*}{$\begin{array}{c}\text { Reporting } \\
\text { by } \\
\text { Glycemic } \\
\text { Control }\end{array}$} & \multirow{2}{*}{\begin{tabular}{|c} 
Regression \\
Analysis on \\
Association \\
Between \\
BMI and \\
Glycemic \\
Control?
\end{tabular}} & \multirow[b]{2}{*}{ Notes } \\
\hline & & & & & $\begin{array}{c}\text { Glycemic } \\
\text { Control Rates } \\
\left(\mathrm{HbA}_{1 \mathrm{C}} \text { Used to }\right. \\
\text { Define Control) }\end{array}$ & $\begin{array}{l}\text { Meana }^{\mathrm{a}} \\
\mathrm{HbA}_{1 \mathrm{c}}\end{array}$ & & & \\
\hline \multicolumn{10}{|c|}{ CHINA } \\
\hline Cai et al [16] & $\begin{array}{c}\text { Observational } \\
\text { prospective } \\
\text { cohort study in } \\
81 \text { hospitals } \\
\text { (Bristol-Myers } \\
\text { Squibb) }\end{array}$ & $\begin{array}{c}\text { Newly } \\
\text { diagnosed } \\
\text { individuals } \\
\text { with T2D } \\
\text { (<6 months) } \\
\mathrm{N}=5770\end{array}$ & $\begin{array}{l}\text { Range of } \\
\text { medi- } \\
\text { cations } \\
\text { (including } \\
\text { oral med- } \\
\text { ications, } \\
\text { herbal } \\
\text { medicine } \\
\text { and insu- } \\
\text { lin) }\end{array}$ & $\begin{array}{l}\text { Study } \\
\text { records }\end{array}$ & $<7 \%$ & & & & \\
\hline & & & & & & & & & \\
\hline
\end{tabular}




\section{Current Research in Diabetes \& Obesity Journal}

\begin{tabular}{|c|c|c|c|c|c|c|c|c|c|}
\hline \multirow{2}{*}{ Reference } & \multirow{2}{*}{$\begin{array}{l}\text { Study Type } \\
\text { (Sponsor) }\end{array}$} & \multirow{2}{*}{$\begin{array}{c}\text { Patient } \\
\text { Population } \\
\text { (Numbers) }\end{array}$} & \multirow{2}{*}{$\begin{array}{l}\text { Treatment } \\
\text { Profile of } \\
\text { Included } \\
\text { Patients }\end{array}$} & \multirow{2}{*}{$\begin{array}{c}\text { Source of } \\
\text { Data for } \\
\text { Weight/ } \\
\text { HbA }_{1 c} \\
\text { Analysis }\end{array}$} & \multicolumn{2}{|c|}{$\begin{array}{l}\text { Reporting by BMI } \\
\text { Category }\end{array}$} & \multirow{2}{*}{$\begin{array}{c}\begin{array}{c}\text { Reporting } \\
\text { by } \\
\text { Glycemic } \\
\text { Control }\end{array} \\
\\
\\
\text { Mean }^{\mathrm{a}} \\
\text { BMI }\end{array}$} & \multirow{2}{*}{\begin{tabular}{|l} 
\\
Regression \\
Analysis on \\
Association \\
Between \\
BMI and \\
Glycemic \\
Control?
\end{tabular}} & \multirow{2}{*}{ Notes } \\
\hline & & & & & $\begin{array}{c}\text { Glycemic } \\
\text { Control Rates } \\
\text { (HbA } A_{11} \text { Used to } \\
\text { Define Control) }\end{array}$ & $\begin{array}{l}\operatorname{Mean}^{\mathrm{a}} \\
\mathrm{HbA}_{1 \mathrm{c}}\end{array}$ & & & \\
\hline $\begin{array}{c}\text { Chen et al } \\
{[10]}\end{array}$ & $\begin{array}{l}\text { Observational } \\
\text { prospective } \\
\text { cross-sectional } \\
\text { study in } 26 \\
\text { medical centers }\end{array}$ & $\begin{array}{l}\text { Adult outpa- } \\
\text { tients with } \\
\text { T2D } \\
\text { N=9065 }\end{array}$ & $\begin{array}{c}\text { Range of } \\
\text { medica- } \\
\text { tions (per- } \\
\text { centage of } \\
\text { patients } \\
\text { on oral } \\
\text { medica- } \\
\text { tions and } \\
\text { insulin } \\
\text { reported, } \\
\text { insulin use } \\
\text { ranged } \\
\text { from } \\
35.05 \% \text { to } \\
52.43 \% \text { of } \\
\text { indi- } \\
\text { viduals, } \\
\text { depending } \\
\text { on BMI } \\
\text { category) }\end{array}$ & $\begin{array}{l}\text { Study } \\
\text { records }\end{array}$ & $\underset{\leq 7 \%}{\checkmark}$ & $\checkmark$ & & $\checkmark$ & \\
\hline Jiet al [11] & $\begin{array}{l}\text { Observational } \\
\text { prospective } \\
\text { cross-sectional } \\
\text { survey study } \\
\text { (Diabetes } \\
\text { Disease Specific } \\
\text { Programme) } \\
\text { (Lilly) }\end{array}$ & $\begin{array}{c}\text { Individuals } \\
\text { with T2D } \\
\text { enrolled in } \\
\text { Diabetes Dis- } \\
\text { ease Specific } \\
\text { Programme } \\
\text { N=2052 }\end{array}$ & $\begin{array}{l}\text { Range of } \\
\text { medi- } \\
\text { cations } \\
(27.9 \% \\
\text { were insu- } \\
\text { lin users) }\end{array}$ & $\begin{array}{c}\text { Survey } \\
\text { (Diabetes } \\
\text { Disease } \\
\text { Specific } \\
\text { Pro- } \\
\text { gramme } \\
\text { - Patient } \\
\text { Record } \\
\text { Form) }\end{array}$ & $\underset{<7 \%}{\checkmark}$ & $\checkmark$ & & $\checkmark$ & \\
\hline Li et al [21] & $\begin{array}{c}\text { Retrospective } \\
\text { cross-sectional } \\
\text { study using } \\
\text { an existing } \\
\text { database in one } \\
\text { tertiary care } \\
\text { center }\end{array}$ & $\begin{array}{l}\text { Individuals } \\
\text { with T2D } \\
\text { receiving } \\
\text { treatment at } \\
\text { the diabetes } \\
\text { center } \\
\mathrm{N}=1387\end{array}$ & 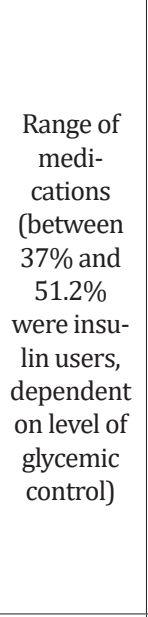 & $\begin{array}{l}\text { Electronic } \\
\text { medical } \\
\text { records }\end{array}$ & & & & $V$ & $\begin{array}{l}\text { Paper reports } \\
\text { categorical BMI } \\
\text { distribution for } \\
\text { controlled and } \\
\text { uncontrolled HbA } \mathrm{HA}_{1 \mathrm{c}} \\
\text { categories (for } \\
\text { uncontrolled or } \\
\text { controlled the pa- } \\
\text { per reports the dis- } \\
\text { tribution across the } \\
\text { BMI categories; e.g. } \\
\text { for the population } \\
\text { of individuals who } \\
\text { are uncontrolled } \\
\text { the paper reports } \\
\text { what \% of that pop- } \\
\text { ulation sits in each } \\
\text { BMI category) }\end{array}$ \\
\hline $\begin{array}{l}\text { Liu et al } \\
\text { [12] }\end{array}$ & $\begin{array}{c}\text { Observational } \\
\text { prospective } \\
\text { cross-sectional } \\
\text { study using } \\
\text { face-to-face } \\
\text { interviews } \\
\text { in } 50 \\
\text { medical cen- } \\
\text { ters }\end{array}$ & $\begin{array}{c}\text { Individuals } \\
\text { with T2D } \\
\text { treated in } \\
\text { medical } \\
\text { centers } \\
\mathrm{N}=5961\end{array}$ & $\begin{array}{l}\text { Range of } \\
\text { medi- } \\
\text { cations } \\
(61.5 \% \\
\text { were } \\
\text { insulin } \\
\text { users) }\end{array}$ & $\begin{array}{l}\text { Electronic } \\
\text { medical } \\
\text { records }\end{array}$ & $\underset{<7 \%}{\checkmark}$ & & & $\checkmark$ & \\
\hline
\end{tabular}




\begin{tabular}{|c|c|c|c|c|c|c|c|c|c|}
\hline \multirow{2}{*}{ Reference } & \multirow{2}{*}{$\begin{array}{l}\text { Study Type } \\
\text { (Sponsor) }\end{array}$} & \multirow{2}{*}{$\begin{array}{l}\text { Patient } \\
\text { Population } \\
\text { (Numbers) }\end{array}$} & \multirow{2}{*}{$\begin{array}{c}\text { Treatment } \\
\text { Profile of } \\
\text { Included } \\
\text { Patients }\end{array}$} & \multirow{2}{*}{$\begin{array}{c}\text { Source of } \\
\text { Data for } \\
\text { Weight/ } \\
\text { HbA }_{1 c} \\
\text { Analysis }\end{array}$} & \multicolumn{2}{|c|}{$\begin{array}{c}\text { Reporting by BMI } \\
\text { Category }\end{array}$} & \multirow{2}{*}{$\begin{array}{c}\begin{array}{c}\text { Reporting } \\
\text { by }\end{array} \\
\text { Glycemic } \\
\text { Control } \\
\\
\begin{array}{c}\text { Mean } \\
\text { BMI }\end{array} \\
\end{array}$} & \multirow{2}{*}{\begin{tabular}{|l|} 
Regression \\
Analysis on \\
Association \\
Between \\
BMI and \\
Glycemic \\
Control?
\end{tabular}} & \multirow{2}{*}{ Notes } \\
\hline & & & & & $\begin{array}{c}\text { Glycemic } \\
\text { Control Rates } \\
\text { (HbA } \text { Hit }_{\text {Used to }} \\
\text { Define Control) }\end{array}$ & $\begin{array}{l}\text { Mean }^{\mathrm{a}} \\
\mathrm{HbA}_{1 \mathrm{c}}\end{array}$ & & & \\
\hline Ma et al [22] & $\begin{array}{l}\text { Retrospective } \\
\text { cohort study in } \\
\text { seven central } \\
\text { hospitals (Jan- } \\
\text { Dec 2010) }\end{array}$ & $\begin{array}{c}\text { Individuals } \\
\text { with T2D } \\
\text { who have } \\
\text { never been } \\
\text { hospitalized } \\
\text { for T2D } \\
\mathrm{N}=17,259\end{array}$ & $\begin{array}{c}\text { Range of } \\
\text { medi- } \\
\text { cations } \\
\text { (6.6\% } \\
\text { were insu- } \\
\text { lin users } \\
\text { in the first } \\
\text { year after } \\
\text { diagnosis. } \\
\text { Data for } \\
\text { this report } \\
\text { were } \\
\text { baseline } \\
\text { data) }\end{array}$ & $\begin{array}{l}\text { Electronic } \\
\text { medical } \\
\text { records }\end{array}$ & $\underset{<7 \%}{\checkmark}$ & $V$ & & & \\
\hline $\begin{array}{l}\text { Wan et al } \\
\text { [23] } \\
\text { Note: study } \\
\text { from Hong } \\
\text { Kong }\end{array}$ & $\begin{array}{l}\text { Retrospective } \\
\text { cohort study in } \\
\text { primary care } \\
\text { patients from } \\
\text { one territory }\end{array}$ & $\begin{array}{l}\text { Individuals } \\
\text { with T2D } \\
\text { in primary } \\
\text { care with no } \\
\text { known CVD } \\
\text { history } \\
\mathrm{N}=115,782\end{array}$ & $\begin{array}{c}\text { Range of } \\
\text { medi- } \\
\text { cations } \\
(1.32 \% \\
\text { were insu- } \\
\text { lin users) }\end{array}$ & $\begin{array}{l}\text { Electronic } \\
\text { medical } \\
\text { records }\end{array}$ & & & $\checkmark$ & & \\
\hline $\begin{array}{l}\text { Wang et al } \\
\text { [13] }\end{array}$ & $\begin{array}{c}\text { Prospective } \\
\text { cross-sectional } \\
\text { questionnaire } \\
\text { study undertak- } \\
\text { en in } 27 \text { centers }\end{array}$ & $\begin{array}{l}\text { Individuals } \\
\text { with T2D } \\
\text { treated with } \\
\text { insulin } \\
\mathrm{N}=2787\end{array}$ & $\begin{array}{c}\text { Insu- } \\
\text { lin-treated } \\
\text { individuals }\end{array}$ & $\begin{array}{l}\text { Study } \\
\text { records }\end{array}$ & & & & $V$ & $\begin{array}{l}\text { Paper reports } \\
\text { categorical BMI } \\
\text { distribution within } \\
\mathrm{HbA}_{11} \text { categories } \\
\text { (in a defined } \mathrm{HbA} \mathrm{A}_{1 c} \\
\text { group the paper } \\
\text { reports distribu- } \\
\text { tion across BMI } \\
\text { categories; e.g. for } \\
\text { the population of } \\
\text { individuals with an } \\
\mathrm{HbA}_{1 \mathrm{c}}<7 \% \text {, the pa- } \\
\text { per reports what } \% \\
\text { of that population } \\
\text { sits within each } \\
\text { BMI category) }\end{array}$ \\
\hline $\begin{array}{l}\text { Zhang et al } \\
\text { [25] }\end{array}$ & $\begin{array}{l}\text { Retrospective } \\
\text { study in a single } \\
\text { hospital }\end{array}$ & $\begin{array}{l}\text { Individuals } \\
\text { with T2D } \\
\mathrm{N}=3224\end{array}$ & $\begin{array}{c}\text { Range of } \\
\text { medi- } \\
\text { cations } \\
(47 \% \\
\text { were insu- } \\
\text { lin users) }\end{array}$ & $\begin{array}{l}\text { Electronic } \\
\text { medical } \\
\text { records }\end{array}$ & & $\checkmark$ & & & \\
\hline Zhu et al [15] & $\begin{array}{l}\text { Prospective } \\
\text { cross-sectional } \\
\text { study in a com- } \\
\text { munity-dwell- } \\
\text { ing elderly } \\
\text { population }\end{array}$ & 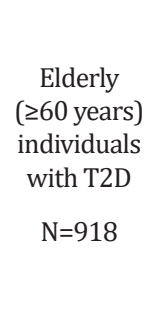 & $\begin{array}{c}\text { Range of } \\
\text { medi- } \\
\text { cation } \\
\text { (82.2\% } \\
\text { to } 88.5 \% \\
\text { were on } \\
\text { anti-dia- } \\
\text { betic med- } \\
\text { ications) }\end{array}$ & $\begin{array}{c}\text { Study } \\
\text { data (local } \\
\text { diabetes } \\
\text { manage- } \\
\text { ment } \\
\text { system) }\end{array}$ & & & $\checkmark$ & & \\
\hline
\end{tabular}




\section{Current Research in Diabetes \& Obesity Journal}

\begin{tabular}{|c|c|c|c|c|c|c|c|c|c|}
\hline \multirow{2}{*}{ Reference } & \multirow{2}{*}{$\begin{array}{l}\text { Study Type } \\
\text { (Sponsor) }\end{array}$} & \multirow{2}{*}{$\begin{array}{l}\text { Patient } \\
\text { Population } \\
\text { (Numbers) }\end{array}$} & \multirow{2}{*}{$\begin{array}{l}\text { Treatment } \\
\text { Profile of } \\
\text { Included } \\
\text { Patients }\end{array}$} & \multirow{2}{*}{$\begin{array}{l}\text { Source of } \\
\text { Data for } \\
\text { Weight/ } \\
\text { HbA }_{1 \mathrm{c}} \\
\text { Analysis }\end{array}$} & \multicolumn{2}{|c|}{$\begin{array}{l}\text { Reporting by BMI } \\
\text { Category }\end{array}$} & \multirow{2}{*}{$\begin{array}{c}\begin{array}{c}\text { Reporting } \\
\text { by } \\
\text { Glycemic } \\
\text { Control }\end{array} \\
\\
\text { Mean }^{\mathrm{a}} \\
\text { BMI }\end{array}$} & \multirow{2}{*}{$\begin{array}{c}\text { Regression } \\
\text { Analysis on } \\
\text { Association } \\
\text { Between } \\
\text { BMI and } \\
\text { Glycemic } \\
\text { Control? }\end{array}$} & \multirow{2}{*}{ Notes } \\
\hline & & & & & $\begin{array}{c}\text { Glycemic } \\
\text { Control Rates } \\
\left(\mathrm{HbA}_{1 \mathrm{c}} \text { Used to }\right. \\
\text { Define Control) }\end{array}$ & $\begin{array}{l}\text { Mean }^{\mathrm{a}} \\
\mathrm{HbA}_{1 \mathrm{c}}\end{array}$ & & & \\
\hline \multicolumn{10}{|c|}{ JAPAN } \\
\hline $\begin{array}{c}\text { Tobe et al } \\
\text { [18] }\end{array}$ & $\begin{array}{c}\text { Prospective } \\
\text { postmar- } \\
\text { keting study } \\
\text { (STELLA-LONG } \\
\text { TERM) of } \\
\text { ipragliflozin } \\
\text { (Astellas) }\end{array}$ & $\begin{array}{l}\text { Individ- } \\
\text { uals with } \\
\text { T2D being } \\
\text { treated with } \\
\text { ipragliflozin } \\
\text { in real life } \\
\mathrm{N}=11,053\end{array}$ & $\begin{array}{l}\text { Ipragli- } \\
\text { flozin } \\
\text { treatment }\end{array}$ & $\begin{array}{l}\text { Study } \\
\text { records }\end{array}$ & $\begin{array}{l}\vee \\
<8 \%\end{array}$ & & & & \\
\hline $\begin{array}{c}\text { Yamakawa et } \\
\text { al [19] }\end{array}$ & $\begin{array}{c}\text { Prospective } \\
\text { multicenter } \\
\text { diary study } \\
\text { (Sleep and } \\
\text { Food Registry) }\end{array}$ & $\begin{array}{l}\text { Individuals } \\
\text { with T2D } \\
\text { participating } \\
\text { in the Sleep } \\
\text { and Food } \\
\text { Registry } \\
\text { in Kanaga- } \\
\text { wa study } \\
\mathrm{N}=3032\end{array}$ & $\begin{array}{l}\text { Range of } \\
\text { medi- } \\
\text { cations } \\
(25.9 \% \\
\text { were insu- } \\
\text { lin users })\end{array}$ & $\begin{array}{l}\text { Study } \\
\text { records }\end{array}$ & & & $\checkmark$ & & \\
\hline $\begin{array}{c}\text { Yokoyama et } \\
\text { al [14] }\end{array}$ & $\begin{array}{l}\text { Prospective } \\
\text { cross-sectional } \\
\text { nationwide } \\
\text { survey }\end{array}$ & $\begin{array}{l}\text { Individuals } \\
\text { with T2D } \\
\text { who attend- } \\
\text { ed primary } \\
\text { care clinics } \\
\mathrm{N}=9956\end{array}$ & $\begin{array}{c}\text { Range of } \\
\text { medi- } \\
\text { cations } \\
(18.6 \% \\
\text { were insu- } \\
\text { lin users) }\end{array}$ & $\begin{array}{l}\text { Study } \\
\text { records }\end{array}$ & $\begin{array}{l}\vee \\
<7 \%\end{array}$ & & & & \\
\hline \multicolumn{10}{|c|}{ SPAIN } \\
\hline $\begin{array}{l}\text { Salinero-Fort } \\
\text { et al [17] }\end{array}$ & $\begin{array}{l}\text { Longitudinal } \\
\text { prospective } \\
\text { outpatient } \\
\text { study (MADIA- } \\
\text { BETES) }\end{array}$ & $\begin{array}{l}\text { T2D individ- } \\
\text { uals } \\
\mathrm{N}=3443\end{array}$ & Not stated & $\begin{array}{l}\text { Study } \\
\text { records }\end{array}$ & $\begin{array}{l}\vee \\
<7 \%\end{array}$ & $V$ & & & \\
\hline \multicolumn{10}{|c|}{ UK } \\
\hline $\begin{array}{c}\text { Aucott et al } \\
{[20]}\end{array}$ & $\begin{array}{c}\text { Retrospective } \\
\text { longitudinal } \\
\text { cohort study, } \\
\text { linking Scottish } \\
\text { Diabetes Care } \\
\text { database re- } \\
\text { cords with hos- } \\
\text { pital admission } \\
\text { and mortality } \\
\text { records }\end{array}$ & $\begin{array}{l}\text { Individuals } \\
\text { overweight/ } \\
\text { obese and } \\
\text { newly } \\
\text { diagnosed } \\
\text { with T2D be- } \\
\text { tween } 2002 \\
\text { and } 2006 \\
\mathrm{~N}=29,316 \text { at } \\
\text { baseline }\end{array}$ & $\begin{array}{l}\text { Range of } \\
\text { medica- } \\
\text { tions }\end{array}$ & $\begin{array}{l}\text { Electronic } \\
\text { medical } \\
\text { records } \\
\text { (Scottish } \\
\text { diabetes } \\
\text { database) }\end{array}$ & & & & $V$ & \\
\hline \multicolumn{10}{|c|}{ USA } \\
\hline Bae et al [2] & $\begin{array}{c}\text { Retrospective } \\
\text { cohort study } \\
\text { across } 38 \text { US } \\
\text { states } \\
\text { (Lilly) }\end{array}$ & $\begin{array}{c}\text { Individuals } \\
\text { diagnosed } \\
\text { with T1D or } \\
\text { T2D } \\
\text { N=248,567 }\end{array}$ & $\begin{array}{l}\text { Range of } \\
\text { medica- } \\
\text { tions }\end{array}$ & $\begin{array}{l}\text { Electronic } \\
\text { medical } \\
\text { records } \\
\text { (Humed- } \\
\text { ica }{ }^{\circledR} \\
\text { database) }\end{array}$ & & & & $\checkmark$ & $\begin{array}{l}\text { Paper reports } \\
\text { categorical BMI } \\
\text { distribution within } \\
\mathrm{HbA}_{1 \mathrm{c}} \text { categories } \\
\text { (in a defined } \mathrm{HbA} \mathrm{A}_{1 \mathrm{c}} \\
\text { group the paper } \\
\text { reports the distri- } \\
\text { bution across the } \\
\text { BMI categories; e.g. } \\
\text { for the population } \\
\text { of individuals with } \\
\text { an } \mathrm{Hb} \mathrm{A}_{1 \mathrm{c}}<7 \% \text {, the } \\
\text { paper reports what } \\
\% \text { of that popu- } \\
\text { lation sits within } \\
\text { each BMI category) }\end{array}$ \\
\hline
\end{tabular}




\section{Current Research in Diabetes \& Obesity Journal}

\begin{tabular}{|c|c|c|c|c|c|c|c|c|c|}
\hline \multirow{2}{*}{ Reference } & \multirow{2}{*}{$\begin{array}{l}\text { Study Type } \\
\text { (Sponsor) }\end{array}$} & \multirow{2}{*}{$\begin{array}{c}\text { Patient } \\
\text { Population } \\
\text { (Numbers) }\end{array}$} & \multirow{2}{*}{$\begin{array}{c}\text { Treatment } \\
\text { Profile of } \\
\text { Included } \\
\text { Patients }\end{array}$} & \multirow{2}{*}{$\begin{array}{l}\text { Source of } \\
\text { Data for } \\
\text { Weight/ } \\
\text { HbA }_{1 \mathrm{c}} \\
\text { Analysis }\end{array}$} & \multicolumn{2}{|c|}{$\begin{array}{l}\text { Reporting by BMI } \\
\text { Category }\end{array}$} & \multirow{2}{*}{$\begin{array}{c}\begin{array}{c}\text { Reporting } \\
\text { by }\end{array} \\
\text { Glycemic } \\
\text { Control } \\
\\
\text { Mean }^{\mathrm{a}} \\
\text { BMI }\end{array}$} & \multirow{2}{*}{$\begin{array}{c}\text { Regression } \\
\text { Analysis on } \\
\text { Association } \\
\text { Between } \\
\text { BMI and } \\
\text { Glycemic } \\
\text { Control? }\end{array}$} & \multirow{2}{*}{ Notes } \\
\hline & & & & & $\begin{array}{c}\text { Glycemic } \\
\text { Control Rates } \\
\text { (HbA Used to } \\
\text { Define Control) }\end{array}$ & $\begin{array}{l}\text { Mean }^{a} \\
\text { HbA }_{1 c}\end{array}$ & & & \\
\hline $\begin{array}{c}\text { Weng et al } \\
\text { [24] }\end{array}$ & $\begin{array}{l}\text { Retrospective } \\
\text { cross-sectional } \\
\text { analysis of } \\
\text { Quintiles da- } \\
\text { tabase (covers } \\
35 \text { million indi- } \\
\text { viduals across } \\
\text { America) } \\
\text { (Novo Nordisk) }\end{array}$ & $\begin{array}{l}\text { Individuals } \\
\text { with T2D, } \\
\text { excluding co- } \\
\text { morbidities } \\
\text { that were } \\
\text { known to } \\
\text { cause exces- } \\
\text { sive weight } \\
\text { changes } \\
\mathrm{N}=414,266 \\
\text { (HbA } 1 \text { data } \\
\text { available } \\
\text { for) }\end{array}$ & $\begin{array}{l}\text { Range of } \\
\text { medi- } \\
\text { cations } \\
(\sim 30 \% \\
\text { were insu- } \\
\text { lin users) }\end{array}$ & $\begin{array}{l}\text { Electronic } \\
\text { medical } \\
\text { records } \\
\text { (Quintiles } \\
\text { database) }\end{array}$ & $\underset{\text { (See note) }}{V}$ & $V$ & & & $\begin{array}{l}\text { Paper reports } \\
\text { categorical } \mathrm{HbA}_{1 \mathrm{c}} \\
\text { distribution within } \\
\text { BMI categories (in a } \\
\text { defined BMI group } \\
\text { the paper reports } \\
\text { the distribution } \\
\text { across the } \mathrm{HbA} \mathrm{ic}_{1 \mathrm{c}} \\
\text { categories; e.g. for } \\
\text { the population of } \\
\text { individuals with } \\
\text { a BMI }<30 \mathrm{~kg} / \mathrm{m}^{2} \text {, } \\
\text { the paper reports } \\
\text { what } \% \text { of that } \\
\text { population sits } \\
\text { within each } \mathrm{HbA} \mathrm{Ac}_{1 \mathrm{c}} \\
\text { category). No sta- } \\
\text { tistics undertaken } \\
\text { in the paper }\end{array}$ \\
\hline $\begin{array}{c}\text { TOTAL } \\
\text { (Number of } \\
\text { studies) }\end{array}$ & & & & & 9 & 6 & 3 & 8 & \\
\hline
\end{tabular}

a Unless stated otherwise.

b We only present $T 2 D$ data.

BMI: body mass index; CVD: cardiovascular disease; HbA1c: glycated hemoglobin; T1D: type 1 diabetes; T2D: type 2 diabetes.

The BMI and the glycemic control categories or ranges used in these studies are presented in Table 3. Importantly, the definition of overweight and obesity differs considerably between Eastern and Western countries. Within specific geographies, there were small inconsistencies with regards to BMI categories. Glycemic control was consistently defined as $<7 \%$, except in two studies $[18,24]$ that used a cut-off of $\mathrm{HbA}_{1 \mathrm{c}}<8 \%$.

Table 3: BMI and glycemic control categories, glycemic control ranges and supporting references.

\begin{tabular}{|c|c|c|c|c|c|}
\hline Reference & BMI Range $\left(\mathrm{kg} / \mathrm{m}^{2}\right)$ & $\begin{array}{l}\text { Reference Source } \\
\text { (BMI) }\end{array}$ & $\begin{array}{c}\text { Glycemic Control } \\
\text { Definition } \\
\text { (Converted to } \\
\mathbf{H b A}_{1 \mathrm{c}} \text { if Needed) }\end{array}$ & $\mathrm{HbA}_{1 \mathrm{c}}$ Ranges & $\begin{array}{l}\text { Reference Source } \\
\text { (Glycemic Control) }\end{array}$ \\
\hline \multicolumn{6}{|c|}{ CHINA } \\
\hline Cai et al [16] & $\begin{array}{c}\text { Normal: }<24 \\
\text { Overweight: } 24 \text { to }<28 \\
\text { Obese: } \geq 28\end{array}$ & $\begin{array}{l}\text { Reference source not } \\
\text { explicitly stated }\end{array}$ & $\mathrm{HbA}_{1 \mathrm{c}}<7 \%$ & NA & $\begin{array}{l}\text { Paper references both ADA (2017) } \\
\text { [26] and Weng et al (2016) [27] }\end{array}$ \\
\hline Chen et al [10] & $\begin{array}{c}\text { Normal: } 18-24 \\
\text { Overweight: } 24-28 \\
\text { Obese: }>28\end{array}$ & $\begin{array}{l}\text { BMI categories refer- } \\
\text { enced to the Chinese } \\
\text { BMI standard (Zhou et } \\
\text { al, 2002) [28] }\end{array}$ & $\mathrm{HbA}_{1 \mathrm{c}} \leq 7 \%$ & NA & $\begin{array}{l}\text { References not given but }<7 \% \\
\text { discussed with mention to 'ADA' and } \\
\text { Chinese glycemia control level }\end{array}$ \\
\hline
\end{tabular}




\section{Current Research in Diabetes \& Obesity Journal}

\begin{tabular}{|c|c|c|c|c|c|}
\hline Reference & BMI Range $\left(\mathrm{kg} / \mathrm{m}^{2}\right)$ & $\begin{array}{l}\text { Reference Source } \\
\text { (BMI) }\end{array}$ & $\begin{array}{l}\text { Glycemic Control } \\
\text { Definition } \\
\text { (Converted to } \\
\text { HbA }_{1 \mathrm{c}} \text { if Needed) }\end{array}$ & $\mathrm{HbA}_{1 \mathrm{c}}$ Ranges & $\begin{array}{l}\text { Reference Source } \\
\text { (Glycemic Control) }\end{array}$ \\
\hline Ji et al [11] & $\begin{array}{c}\text { Underweight: }<18.5 \\
\text { Normal: } 18.5 \text { to }<24.0 \\
\text { Overweight: } 24.0 \text { to }<28.0 \\
\text { Obese } \geq 28.0\end{array}$ & $\begin{array}{l}\text { BMI categories en- } \\
\text { dorsed by the National } \\
\text { Health \& Family Plan- } \\
\text { ning Commission of the } \\
\text { People's Republic of } \\
\text { China (2013) [29] }\end{array}$ & $\begin{array}{c}\mathrm{HbA}_{1 \mathrm{c}}<7 \% \\
\text { Also provides data } \\
\text { for } \mathrm{HbA}_{1 \mathrm{c}} \geq 9 \%\end{array}$ & NA & References not given \\
\hline Li et al [21] & $\begin{array}{c}\text { Underweight: }<18.5 \\
\text { Normal: } 18.5-23.9 \\
\text { Overweight: } 24.0-27.9 \\
\text { Obese: } \geq 28\end{array}$ & $\begin{array}{l}\text { BMI categories ref- } \\
\text { erenced to the China } \\
\text { Expert Panel of Medical } \\
\text { Nutrition Therapy for } \\
\text { Overweight/Obesity. } \\
\text { Expert consensus on } \\
\text { medical nutrition thera- } \\
\text { py for overweight/obe- } \\
\text { sity in China (2016) }\end{array}$ & $\mathrm{HbA}_{1 \mathrm{c}}<7 \%$ & NA & Chinese Diabetes Society (2014) [30] \\
\hline Liu et al [12] & $\begin{array}{c}\text { Normal: } 18.5-23.9 \\
\text { Overweight: } 24.0-27.9 \\
\text { Obese: } \geq 28\end{array}$ & $\begin{array}{l}\text { BMI categories refer- } \\
\text { enced to the Chinese } \\
\text { Medical Association. } \\
\text { Expert Consensus on } \\
\text { Chinese adult obesity } \\
\text { prevention (2011) }\end{array}$ & $\mathrm{HbA}_{1 c}<7 \%$ & NA & ADA (2015) [31] \\
\hline Ma et al [22] & $\begin{array}{c}\text { Underweight: }<18.5 \\
\text { Normal: } 18.5-23.99 \\
\text { Overweight: } 24.0-27.99 \\
\text { Obese: } \geq 28.0\end{array}$ & $\begin{array}{l}\text { BMI categories refer- } \\
\text { enced to The Depart- } \\
\text { ment of Disease Control } \\
\text { Ministry of Health PRC. } \\
\text { The guidelines for pre- } \\
\text { vention and control of } \\
\text { overweight and obesity } \\
\text { in Chinese adults }\end{array}$ & $\begin{array}{c}<53 \mathrm{mmol} / \mathrm{mol} \\
\text { (equivalent to } \\
\mathrm{HbA}_{1 \mathrm{c}}<7 \% \text { ) }\end{array}$ & NA & References not given \\
\hline Wan et al [23] & NA & NA & NA & $\begin{array}{c}<6 \% \\
6-6.4 \% \\
6.5-6.9 \% \\
7-7.4 \% \\
7.5-7.9 \% \\
8-8.4 \% \\
8.5-8.9 \% \\
9-9.4 \% \\
9.5-9.9 \% \\
\geq 10 \%\end{array}$ & $\begin{array}{l}\text { Authors state: 'Most international } \\
\text { guidelines provide a recommended } \\
\text { optimal } \mathrm{HbA}_{1 \mathrm{c}} \text { target as the goal } \\
\text { for diabetic management. How- } \\
\text { ever, there is no clearly apparent } \\
\text { consensus on the optimal HbA } \\
\text { target, which can vary from }<6.5 \% \text { to } \\
<8.0 \% \text {. Recent guidelines including } \\
\text { the American Diabetes Association } \\
\text { and European Association for the } \\
\text { Study of Diabetes are now advocating } \\
\text { replacing rigid and uniform targets } \\
\text { to one which is more nuanced and } \\
\text { patient-centered, however more ev- } \\
\text { idence is still needed to support the } \\
\text { call for individualized } \mathrm{HbA}_{1 \mathrm{c}} \text { targets' }\end{array}$ \\
\hline $\begin{array}{l}\text { Wang et al } \\
\text { [13] }\end{array}$ & $\begin{array}{c}\text { Underweight: }<18.5 \\
\text { Normal: } 18.5 \text { to }<24.0 \\
\text { Overweight: } 24.0 \text { to }<28.0 \\
\text { Obese: } \geq 28.0\end{array}$ & $\begin{array}{l}\text { Reference source not } \\
\text { explicitly stated }\end{array}$ & $\mathrm{HbA}_{1 \mathrm{c}}<7 \%$ & & $\begin{array}{l}\text { Diabetes Society of the Chinese } \\
\text { Medical Association (2018) [32] }\end{array}$ \\
\hline
\end{tabular}




\section{Current Research in Diabetes \& Obesity Journal}

\begin{tabular}{|c|c|c|c|c|c|}
\hline Reference & BMI Range $\left(\mathrm{kg} / \mathrm{m}^{2}\right)$ & $\begin{array}{l}\text { Reference Source } \\
\text { (BMI) }\end{array}$ & $\begin{array}{l}\text { Glycemic Control } \\
\text { Definition } \\
\text { (Converted to } \\
\text { HbA }_{1 \mathrm{c}} \text { if Needed) }\end{array}$ & $\mathrm{HbA}_{1 \mathrm{c}}$ Ranges & $\begin{array}{l}\text { Reference Source } \\
\text { (Glycemic Control) }\end{array}$ \\
\hline $\begin{array}{l}\text { Zhang et al } \\
{[25]}\end{array}$ & $\begin{array}{l}\text { BMI quintiles: } \\
\quad<21.62 \\
21.62-23.50 \\
23.51-25.16 \\
25.17-27.33 \\
>27.33\end{array}$ & NA & NA & NA & NA \\
\hline Zhu et al [15] & NA & NA & $\mathrm{HbA}_{1 \mathrm{c}} \leq 7 \%$ & NA & $\begin{array}{c}\text { References two papers: Li et al } \\
\text { (2018) [21] and Yang et al (2016) } \\
\text { [33] }\end{array}$ \\
\hline \multicolumn{6}{|c|}{ JAPAN } \\
\hline Tobe et al [18] & $\begin{array}{c}\quad<22.0 \\
22.0 \text { to }<25.0 \\
25.0 \text { to }<30.0 \\
\geq 30.0 \\
\text { Note: Label not attached } \\
\text { to each category }\end{array}$ & $\begin{array}{l}\text { Japanese Society for } \\
\text { the Study of Obesity } \\
\text { Diseases (2016) [34] }\end{array}$ & $\mathrm{HbA}_{1 \mathrm{c}}<8 \%$ & NA & Reference source not explicitly stated \\
\hline $\begin{array}{l}\text { Yamakawa et } \\
\text { al [19] }\end{array}$ & NA & NA & NA & $\begin{array}{c}<6.5 \% \\
6.5-7.0 \% \\
7.0-7.5 \% \\
7.5-8.0 \% \\
>8.0 \%\end{array}$ & Reference source not explicitly stated \\
\hline $\begin{array}{l}\text { Yokoyama et } \\
\text { al [14] }\end{array}$ & $\begin{array}{c}<25.0 \\
25 \text { to }<30 \\
\geq 30.0\end{array}$ & $\begin{array}{l}\text { Reference source not } \\
\text { explicitly stated }\end{array}$ & $\mathrm{HbA}_{1 \mathrm{c}}<7 \%$ & NA & Haneda et al (2018) [35] \\
\hline \multicolumn{6}{|c|}{ SPAIN } \\
\hline $\begin{array}{l}\text { Salinero-Fort } \\
\text { et al [17] }\end{array}$ & $\begin{array}{l}\text { Reference categories } \\
\text { based on distribution of } \\
\text { the data by percentiles } \\
\text { P5: }<23.0 \\
\text { P5-25: } 23.0-26.8 \\
\text { P25-75: 26.9-33.1 } \\
\text { P75-95: 33.2-39.4 } \\
\text { P>95: >39.4 }\end{array}$ & NA & $\mathrm{HbA}_{1 \mathrm{c}}<7 \%$ & NA & ADA (2011) [36] \\
\hline \multicolumn{6}{|c|}{ UK } \\
\hline $\begin{array}{c}\text { Aucott et al } \\
{[20]}\end{array}$ & $\begin{array}{c}25-29.9 \\
30-34.9 \\
35-39.9 \\
\geq 40\end{array}$ & $\begin{array}{l}\text { Reference source not } \\
\text { explicitly stated }\end{array}$ & $\mathrm{HbA}_{1 \mathrm{c}} \leq 7 \%$ & NA & $\begin{array}{l}\text { Authors state: 'Glycaemic control } \\
\text { definitions vary. While }<48 \text { mmol/ } \\
\text { mol (or 6.5\%) is an absolute target, } \\
\text { we defined "control" as } \mathrm{HbA}_{1 \mathrm{c}} \leq 53 \\
\text { mmol/mol (or 7\%), a commonly } \\
\text { used clinical classification'. They ref- } \\
\text { erence two papers: Chiu et al (2013) } \\
\text { [37] and Berkowitz et al (2014) [38] }\end{array}$ \\
\hline \multicolumn{6}{|c|}{ USA } \\
\hline
\end{tabular}




\section{Current Research in Diabetes \& Obesity Journal}

\begin{tabular}{|c|c|c|c|c|c|}
\hline Reference & BMI Range $\left(\mathrm{kg} / \mathrm{m}^{2}\right)$ & $\begin{array}{l}\text { Reference Source } \\
\text { (BMI) }\end{array}$ & $\begin{array}{l}\text { Glycemic Control } \\
\text { Definition } \\
\text { (Converted to } \\
\text { HbA }_{1 \mathrm{c}} \text { if Needed) }\end{array}$ & $\mathrm{HbA}_{1 \mathrm{c}}$ Ranges & $\begin{array}{l}\text { Reference Source } \\
\text { (Glycemic Control) }\end{array}$ \\
\hline Bae et al [2] & $\begin{array}{c}\text { Normal: } 18.5 \text { to }<25 \\
\text { Overweight: } 25 \text { to }<30 \\
\text { Obesity I: } 30 \text { to }<35 \\
\text { Obesity II: } 35 \text { to }<40 \\
\text { Obesity III: } \geq 40\end{array}$ & $\begin{array}{l}\text { WHO (2015), BMI } \\
\text { classification [39] }\end{array}$ & NA & $\begin{array}{c}<7 \% \\
\geq 7 \text { to }<8 \% \\
\geq 8 \text { to }<9 \% \\
\geq 9 \%\end{array}$ & $\begin{array}{l}\text { Authors note that glycemic control } \\
\text { cut-offs were based on clinical } \\
\text { guidelines that recommend a treat- } \\
\text { ment target of } \mathrm{HbA}_{1 \mathrm{c}}<7 \% \text { for many } \\
\text { non-pregnant adults with diabetes } \\
\text { and suggest that a target of }<8 \% \\
\text { may be appropriate for patients with } \\
\text { a history of severe hypoglycemia, } \\
\text { limited life expectancy, or diabetic } \\
\text { complications or comorbidities [31]. } \\
\text { Furthermore, results from an ob- } \\
\text { servational study among the elderly } \\
\text { suggest that a target } \mathrm{HbA} \mathrm{A}_{\mathrm{c}} \text { of } 8-8.9 \% \\
\text { may be appropriate [40] and that } \\
\text { an HbA } \mathrm{Hb} \text { value of }>9 \% \text { is associated } \\
\text { with increased mortality risk among } \\
\text { patients with type } 2 \text { diabetes [41] }\end{array}$ \\
\hline $\begin{array}{l}\text { Weng et al } \\
\text { [24] }\end{array}$ & $\begin{array}{c}\quad<30 \\
30 \text { to }<35 \\
35 \text { to }<40 \\
\geq 40 \\
\begin{array}{c}\text { Note: Label not attached } \\
\text { to each category }\end{array}\end{array}$ & $\begin{array}{l}\text { Reference source not } \\
\text { explicitly stated }\end{array}$ & NA & $\begin{array}{c}\leq 6.5 \\
>6.5 \% \text { to }<8 \% \\
\quad \geq 8 \% \\
\quad \geq 9 \% \\
\text { Note: } \text { The } \geq 8 \% \\
\text { includes the } \\
\geq 9 \% \text { category }\end{array}$ & $\begin{array}{c}\text { Authors state that } \geq 8 \% \text { is consid- } \\
\text { ered a lower limit of poor glycemic } \\
\text { control, patient BMI distributions } \\
\text { within the categories of } \mathrm{HbA}_{1 \mathrm{c}} \geq 8 \% \\
\text { and } \geq 9 \% \text { was more closely examined } \\
\text { to determine whether a relationship } \\
\text { between glycemic control and BMI } \\
\text { could be ascertained. } \leq 6.5 \% \text { is con- } \\
\text { sidered to represent good glycemic } \\
\text { control }\end{array}$ \\
\hline
\end{tabular}

ADA: American Diabetes Association; BMI: body mass index; HbA1c: glycated hemoglobin; NA: not applicable; P: percentile; WHO: World Health Organization.

\section{Patient characteristics}

The included studies varied in terms of population numbers and individual characteristics. Population numbers ranged from 918 in a study of community-dwelling elderly people with T2D [15] to 414,266 in the Quintiles EMR database [24].

Some of the study populations were relatively specific and focused - for example, the study by Zhu et al. [15] was undertaken in elderly adults aged $\geq 60$ years and the study by Cai et al. [16] was in newly diagnosed individuals. Other studies were much broader - for example, Bae et al. [2] had few exclusion criteria; data were sourced from EMRs from the Humedica database, which comes from a US network of provider organizations that treat $\sim 30$ million individuals in 38 states who may be uninsured or insured via commercial insurance, Medicare, or Medicaid.

In those studies that reported gender across the whole cohort, approximately 50\% were male (range, 45.31-55.70\%). There was little age differentiation between studies: where an overall figure was given, mean age ranged from 55.7 to 65.0 years. Mean BMI was reported in around half the studies. There were clear differences between geographic regions, with BMI levels in China and Japan lower than in Western countries. For example, in the largest US study $(\mathrm{N}=626,386)[24]$, the mean BMI was $33.2 \mathrm{~kg} / \mathrm{m}^{2}$, compared with the largest ( $\mathrm{N}=115,782)$ study from Asia (Hong Kong) [23], where mean BMI was $25.57 \mathrm{~kg} / \mathrm{m}^{2}$.

The use of anti-diabetic medications varied across studies. Most of the studies (15 out of 17) included individuals on a wide range of therapies. The proportion of individuals on the different medications varied across studies, from $1.32 \%$ [23] to over $85 \%$ [15].

\section{Study findings}

\section{Reported by BMI category}

\section{Glycemic control rates}

Studies either reporting glycemic control rates by BMI category or where BMI was explored as a factor in regression analysis for poor glycemic control are summarized in Table 4 \& Figure 2. Eight $[2,10,12,14,17,18,20,22]$ of the 13 studies that focused on achieving glycemic control (generally defined as $\mathrm{HbA}_{1 \mathrm{c}}$ $<7 \%$ ) reported by BMI category demonstrated that the percentage of patients achieving glycemic control significantly declined as BMI increased, and/or that the likelihood of achieving glycemic control was lower. Just one study focused explicitly on association between BMI and glycemic control, noting that little research had previously explored this in a large, insured US patient population. 


\section{Current Research in Diabetes \& Obesity Journal}

In this study, multinomial logistic regressions examined the association while controlling for a range of confounders including patient demographics, general health, comorbid conditions, and anti-hyperglycemic medication use. The authors reported that for T2D patients, there were positive and statistically significant associations between being overweight or obese and having suboptimal glycemic control. For example, individuals in obesity class III were found to have a $37 \%$ increase in the probability of having an $\mathrm{HbA}_{1 \mathrm{c}} \geq 7 \%$ and $<8 \%$, a $60 \%$ increase in the probability of having an $\mathrm{HbA}_{1 \mathrm{c}} \geq 8 \%$ and $<9 \%$, and a $62 \%$ increase in the probability of having an $\mathrm{HbA}_{1 \mathrm{c}} \geq 9 \%$ [2].

Table 4: Studies reporting glycemic control rates by BMI, and BMI as a factor in regression analyses for poor glycemic control.

\begin{tabular}{|c|c|c|c|}
\hline \multirow{2}{*}{ Reference } & \multirow{2}{*}{$\mathbf{N}$} & \multicolumn{2}{|c|}{ Findings } \\
\hline & & Glycemic Control Rates Reported by BMI & $\begin{array}{l}\text { Regression Analyses Exploring BMI as a Factor } \\
\text { for Poor Glycemic Control }\end{array}$ \\
\hline \multicolumn{4}{|c|}{ Studies showing that glycemic control worsens with increasing BMI (statistically significant) } \\
\hline $\begin{array}{l}\text { Aucott et al } \\
\text { (UK) [20] }\end{array}$ & $\begin{array}{l}15,757 \text { (at Year 1) to } 12,401 \\
\text { (at Year 5) }\end{array}$ & & $\begin{array}{l}\text { Higher diagnosis BMI was one of the factors associ- } \\
\text { ated with poor glycemic control }\left(\mathrm{HbA}_{1 \mathrm{c}}>7 \%\right) \\
\text { Significantly higher risk of not being in control for } \\
\text { each increasing BMI category, vs being over- } \\
\text { weight }\left(25-29.9 \mathrm{~kg} / \mathrm{m}^{2}\right) \text { at } 1 \text {-year follow-up. } \\
\text { For example, the relative risk for not achieving } \\
\text { glycemic control at Year } 1\left(\mathrm{HbA}_{1 \mathrm{c}}>7 \%\right) \text { was } 1.17 \\
(95 \% \mathrm{CI}, 1.09-1.26) \text { for someone with a BMI of } \\
30-34.9 \mathrm{~kg} / \mathrm{m}^{2} \mathrm{vs} 25-29.9 \mathrm{~kg} / \mathrm{m}^{2}(\mathrm{p}<0.001) \\
\text { Significant associations were also shown at } 2-, 3-\text {, } \\
\text { and 5-year follow-up }\end{array}$ \\
\hline $\begin{array}{l}\text { Bae et al } \\
\text { (USA) [2] }\end{array}$ & 248,567 & & 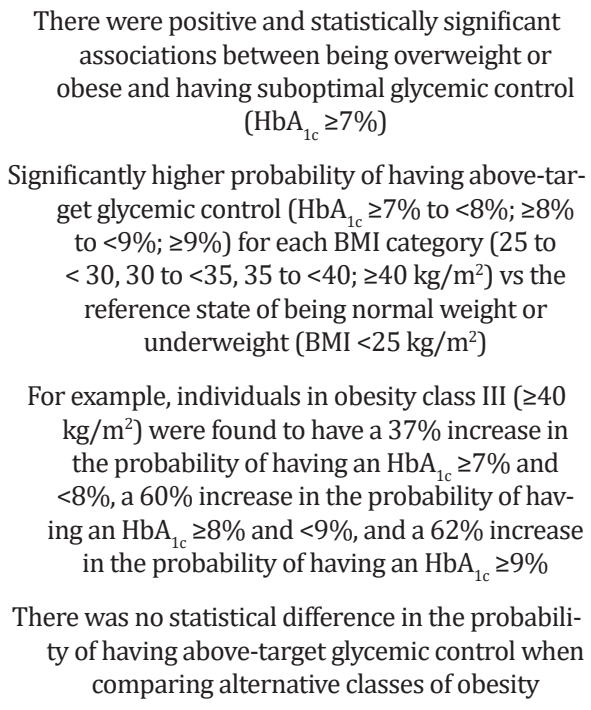 \\
\hline $\begin{array}{l}\text { Chen et al } \\
\text { (China) [10] }\end{array}$ & 9065 & $\begin{array}{l}\text { Glycemic control rates differed among BMI } \\
\text { groups, with } 33.7 \% \text { of individuals in the }<24 \\
\mathrm{~kg} / \mathrm{m}^{2} \text { group, } 33.8 \% \text { in the } 24-28 \mathrm{~kg} / \mathrm{m}^{2} \\
\text { group, and } 30.2 \% \text { in the }>28 \mathrm{~kg} / \mathrm{m}^{2} \text { group } \\
\text { having an } \mathrm{HbA}_{1 \mathrm{c}} \text { of } \leq 7 \%(\mathrm{p}=0.005) \\
\text { This held even after partial correction analysis } \\
\text { adjusting for age, degree of education, dura- } \\
\text { tion of diabetes, } \mathrm{HbA}_{1 \mathrm{c}^{\prime}} \text { and } \mathrm{FPG}(\mathrm{p}=0.006)\end{array}$ & $\begin{array}{l}\text { Higher BMI reported to be an independent risk fac- } \\
\text { tor for poor glycemic control defined as } \mathrm{HbA}_{1 \mathrm{c}} \\
\quad>7 \%(\mathrm{p}=0.026 \text { in multivariate analysis })\end{array}$ \\
\hline
\end{tabular}




\section{Current Research in Diabetes \& Obesity Journal}

\begin{tabular}{|c|c|c|c|}
\hline \multirow[b]{2}{*}{ Reference } & \multirow[b]{2}{*}{$\mathbf{N}$} & \multicolumn{2}{|c|}{ Findings } \\
\hline & & Glycemic Control Rates Reported by BMI & $\begin{array}{l}\text { Regression Analyses Exploring BMI as a Factor } \\
\text { for Poor Glycemic Control }\end{array}$ \\
\hline $\begin{array}{l}\text { Liu et al } \\
\text { (China) [12] }\end{array}$ & 5961 & $\begin{array}{l}\text { Individuals with lower levels of BMI had signif- } \\
\text { icantly }(\mathrm{p}<0.01) \text { higher rates of achieving } \\
\text { glycemic control }\left(\mathrm{HbA}_{1 \mathrm{c}}<7 \%\right) \\
\text { The percentage of patients achieving control } \\
\left(\mathrm{HbA}_{1 \mathrm{c}}<7 \%\right) \text { who were classified as normal, } \\
\text { overweight, or obese was } 36.4 \%, 31.3 \% \text {, and } \\
26.7 \% \text {, respectively }\end{array}$ & $\begin{array}{l}\text { BMI reported to be one of the factors associated } \\
\text { with attainment of } \mathrm{HbA}_{1 \mathrm{c}} \text { (and blood pressure } \\
\text { and cholesterol goals) (OR 0.584; } \mathrm{p}=0.001)\end{array}$ \\
\hline $\begin{array}{l}\text { Ma et al } \\
\text { (China) [22] }\end{array}$ & 17,259 & $\begin{array}{c}\text { The proportion of individuals achieving glyce- } \\
\text { mic control }\left(\mathrm{HbA}_{1 \mathrm{c}}<7 \% \text { ) was significantly }\right. \\
(\mathrm{p}<0.001) \text { lower in individuals with obesity or } \\
\text { overweight vs individuals of normal weight } \\
\text { The percentage of patients achieving control } \\
\left(\mathrm{HbA}_{1 \mathrm{c}}<7 \% \text { ) who were classified as normal, }\right. \\
\text { overweight, or obese was } 58.6 \%, 50.7 \% \text {, and } \\
45.5 \% \text {, respectively }\end{array}$ & \\
\hline $\begin{array}{c}\text { Salinero-Fort } \\
\text { et al (Spain) } \\
\text { [17] }\end{array}$ & 3443 & $\begin{array}{l}\text { The percentage of individuals achieving glycemic } \\
\text { control }\left(\mathrm{HbA}_{\mathrm{c}}<7 \%\right) \text { reduced significantly } \\
(\mathrm{p}=0.001) \text { with higher levels of BMI } \\
\text { The percentage of patients achieving control } \\
\text { ranged from } 58.1 \% \text { in the } 5 \text { th percentile to } \\
45.5 \% \text { in the } 95 \text { th percentile }\end{array}$ & \\
\hline $\begin{array}{l}\text { Tobe et al } \\
\text { (Japan) [18] }\end{array}$ & 11,053 & $\begin{array}{l}\text { As BMI increased, the proportion of individuals } \\
\text { achieving glycemic control }\left(\mathrm{HbA}_{1 \mathrm{c}}<8 \%\right) \text { was } \\
\text { significantly reduced }(\mathrm{p}<0.001) \\
\text { The percentage of patients achieving control } \\
\left(\mathrm{HbA}_{1 \mathrm{c}}<8 \%\right) \text { ranged from } 58.9 \% \text { in those with } \\
\text { a BMI }<22 \mathrm{~kg} / \mathrm{m}^{2} \text { to } 51.7 \% \text { in those with a BMI } \\
\geq 30 \mathrm{~kg} / \mathrm{m}^{2}\end{array}$ & \\
\hline $\begin{array}{l}\text { Yokoyama et al } \\
\text { (Japan) [14] }\end{array}$ & 9956 & $\begin{array}{l}\text { Higher BMI levels were associated with reduction } \\
\text { of the rates of achieving therapeutic } \mathrm{HbA}_{1 \mathrm{c}} \\
\text { target of }<7 \% \text {. The authors concluded that in- } \\
\text { creasing BMI levels correlated with decreasing } \\
\text { rates of achieving all targets (p<0.001); } \mathrm{HbA}_{1 \mathrm{c}} \\
\text { was one of these targets (along with blood } \\
\text { pressure and lipids) } \\
\text { The percentage of individuals achieving control } \\
(<7 \% \text { ) was } 57.1 \% \text { those with a BMI }<25 \mathrm{~kg} / \\
\mathrm{m}^{2}, 48.6 \% \text { in those with a BMI } 25 \text { to }<30 \mathrm{~kg} / \\
\mathrm{m}^{2} \text {, and } 44.7 \% \text { in those with a BMI } \geq 30 \mathrm{~kg} / \mathrm{m}^{2}\end{array}$ & \\
\hline
\end{tabular}




\begin{tabular}{|c|c|c|c|}
\hline \multirow{2}{*}{ Reference } & \multirow{2}{*}{$\mathbf{N}$} & \multicolumn{2}{|c|}{ Findings } \\
\hline & & Glycemic Control Rates Reported by BMI & $\begin{array}{l}\text { Regression Analyses Exploring BMI as a Factor } \\
\text { for Poor Glycemic Control }\end{array}$ \\
\hline \multicolumn{4}{|c|}{ Studies showing a trend of glycemic control worsening with increasing BMI } \\
\hline $\begin{array}{l}\text { Weng et al } \\
\text { (USA) [24] }\end{array}$ & 626,386 & $\begin{array}{l}\text { The prevalence of poor glycemic control increases } \\
\text { as BMI increases } \\
\text { The proportion of patients in the category of good } \\
\text { glycemic control }\left(\mathrm{HbA}_{1 \mathrm{c}} \leq 6.5 \% \text { ) decreased as }\right. \\
\text { BMI category increased. In those individuals } \\
\text { with a BMI }<30 \mathrm{~kg} / \mathrm{m}^{2} \text {, it was reported that } \\
40.1 \% \text { had an } \mathrm{HbA} \mathrm{A}_{1 \mathrm{c}} \leq 6.5 \% \text {; this compared } \\
\text { with } 30.1 \% \text { of individuals with a BMI } \geq 40 \mathrm{~kg} / \\
\mathrm{m}^{2} \\
\text { Conversely, the proportions of patients with poor } \\
\text { glycemic control }\left(\mathrm{HbA}_{1 \mathrm{c}} \geq 8 \% \text { ) increased with }\right. \\
\text { increasing BMI category. In those individuals } \\
\text { with a BMI }<30 \mathrm{~kg} / \mathrm{m}^{2} \text {, it was reported that } \\
21 \% \text { had an } \mathrm{HbA} \mathrm{A}_{1 \mathrm{c}} \geq 8 \% \text {; this compared with } \\
30.2 \% \text { of individuals with a BMI } \geq 40 \mathrm{~kg} / \mathrm{m}^{2} \\
\text { No statistical tests for differences were used to } \\
\text { differentiate } \\
\text { among patients in the four BMI categories }\end{array}$ & \\
\hline \multicolumn{4}{|c|}{ Studies showing some evidence that some statistically significant evidence that glycemic control worsens with higher levels of BMI } \\
\hline $\begin{array}{l}\text { Cai et al } \\
\text { (China) [16] }\end{array}$ & 5770 & $\begin{array}{l}\text { At baseline, the percentage of individuals achiev- } \\
\text { ing glycemic control }\left(\mathrm{HbA}_{1 \mathrm{c}}<7 \%\right) \text { with a BMI } \\
\text { of }<24,24 \text { to }<28 \text {, and } \geq 28 \mathrm{~kg} / \mathrm{m}^{2} \text { was } 35.7 \% \text {, } \\
39.1 \% \text {, and } 33.7 \% \text {, respectively } \\
\text { At } 1 \text { year, the percentage of individuals achieving } \\
\text { glycemic control with a BMI of }<24,24 \text { to }<28 \text {, } \\
\text { and } \geq 28 \mathrm{~kg} / \mathrm{m}^{2} \text { was } 70.3 \%, 69.2 \% \text {, and } 62.0 \% \text {, } \\
\text { respectively }\end{array}$ & $\begin{array}{c}\text { Patients with obesity were found in the multivari- } \\
\text { ate model to have a significantly higher proba- } \\
\text { bility (RR } 1.05 ; \mathrm{p}=0.0044 \text { ) of failing to achieve } \\
\text { glycemic control ( } \mathrm{HbA}_{1 \mathrm{c}}<7 \% \text { ) than individuals } \\
\text { of normal weight (in overweight individuals, the } \\
\text { RR was not significant) at 1-year follow-up } \\
\text { The global p-value (for overweight/obesity vs } \\
\text { normal) was significant ( } \mathrm{p}=0.02 \text { ) }\end{array}$ \\
\hline $\begin{array}{l}\text { Ji et al } \\
\text { (China) [11] }\end{array}$ & 2052 & $\begin{array}{l}\text { With regards to an } \mathrm{HbA}_{1 \mathrm{c}} \text { target of }<7.0 \% \text {, it was } \\
\text { found (using unadjusted analyses) that sig- } \\
\text { nificantly more individuals in the overweight } \\
\text { group than in the obese group achieved an } \\
\mathrm{Hb} \mathrm{A}_{1 \mathrm{c}} \text { target of }<7.0 \% \text { ( } 39.8 \% \text { vs } 27.3 \% \text {; } \\
\mathrm{p}=0.001 \text { ). Also, significantly fewer individ- } \\
\text { uals in the normal BMI group than in the } \\
\text { overweight group achieved an } \mathrm{HbA}_{1 \mathrm{c}} \text { target of } \\
<7.0 \% \text { ( } 33.3 \% \text { vs } 39.8 \% \text {; }=0.006 \text { ) } \\
\text { When looking at the population with an } \mathrm{Hb} \mathrm{A}_{1 \mathrm{c}} \\
\geq 9.0 \% \text {, it was reported that significantly fewer } \\
\text { individuals in the normal BMI group than } \\
\text { the obese group had an } \mathrm{Hb} \mathrm{A}_{1 \mathrm{c}} \geq 9.0 \% \text { ( } 4.3 \% \\
\text { vs } 10.2 \% \text {; } \mathrm{p}=0.002 \text { ). In addition, significantly } \\
\text { more individuals in the obese group had an } \\
\mathrm{HbA}_{1 \mathrm{c}} \geq 9.0 \% \text { vs the overweight group (10.2\% } \\
\text { vs } 5.5 \% \text {; }=0.024 \text { ) }\end{array}$ & $\begin{array}{l}\text { Using adjusted methods, the results were not fully } \\
\text { confirmed }\end{array}$ \\
\hline \multicolumn{4}{|c|}{ Studies that did not show that glycemic control worsens with increasing BMI (statistically significant) } \\
\hline $\begin{array}{l}\text { Li et al } \\
\text { (China) [21] }\end{array}$ & 1387 & & $\begin{array}{c}\text { Simple logistic regression analysis found no be- } \\
\text { tween-BMI group differences in the achieve- } \\
\text { ment of } \mathrm{HbA}_{1 \mathrm{c}}<7.0 \%(\mathrm{p}=0.817)\end{array}$ \\
\hline $\begin{array}{l}\text { Wang et al } \\
\text { (China) [13] }\end{array}$ & 2787 & & $\begin{array}{l}\text { The standard used in this paper for a statistically } \\
\text { significant result appears to be } \mathrm{p}<0.0001 \text {, with } \\
\text { a p-value of } 0.0133 \text { for BMI; this is interpreted } \\
\text { here to mean that the relationship between BMI } \\
\text { and glycemic control was not sufficiently clear } \\
\text { to warrant further investigation using machine } \\
\text { learning techniques }\end{array}$ \\
\hline
\end{tabular}




\section{Current Research in Diabetes \& Obesity Journal}

The largest study included in this review $(\mathrm{N}=626,386)$ [24] did not undertake any statistical testing to differentiate among the four BMI categories, as the analysis was intended to be exploratory. In those individuals with a $\mathrm{BMI}<30 \mathrm{~kg} / \mathrm{m}^{2}$, it was reported that $40.1 \%$ had good glycemic control $\left(\mathrm{HbA}_{1 \mathrm{c}} \leq 6.5 \%\right)$, compared with $30.1 \%$ of individuals with a BMI $\geq 40 \mathrm{~kg} / \mathrm{m}^{2}$. Conversely, the proportions of patients with poor glycemic control $\left(\mathrm{HbA}_{1 \mathrm{c}} \geq 8 \%\right)$ increased with increasing BMI category. In those individuals with a $\mathrm{BMI}<30 \mathrm{~kg} / \mathrm{m}^{2}$, it was reported that $21 \%$ had an $\mathrm{HbA}_{1 \mathrm{c}} \geq 8 \%$, compared with $30.2 \%$ of individuals with a BMI $\geq 40 \mathrm{~kg} / \mathrm{m}^{2}$.

Two studies reported some evidence that higher levels of BMI were associated with significantly worse glycemic control: one study [16] found that individuals with obesity had a significantly higher probability of failing to achieve glycemic control than those of normal weight, but in those who were overweight the probability was not significant. The global p-value for the combined overweight/obesity group compared to normal was significant. The other study [11] found that the use of adjusted methods did not fully confirm the findings from the unadjusted analyses.

Li et al. [21] did not report any significant between-BMI group differences in the achievement of glycemic control ( $\left.\mathrm{HbA}_{1 \mathrm{c}}<7.0 \%\right)$, and the relationship between BMI and glycemic control was not significant in the study by Wang et al. [13].

\section{Mean $\mathrm{HbA}_{1 \text { c }}$}

Four of six studies that reported average levels of $\mathrm{HbA}_{1 \mathrm{c}}$ reported them to be greater in patients with higher BMI (Table 5). In one of these studies, it should be noted that no statistical analysis was undertaken [24], while in another, the mean $\mathrm{HbA}_{1 \mathrm{c}}$ in the lowest 5th BMI percentile was slightly higher than the mean $\mathrm{HbA}_{1 \mathrm{c}}$ for the 25th BMI percentile, but otherwise $\mathrm{HbA}_{1 \mathrm{c}}$ increased as BMI increased [17]. In this study when median $\mathrm{HbA}_{1 \mathrm{c}}$ values were used, the values increased with BMI percentiles consistently across all percentiles. In two studies $[20,25]$, the mean $\mathrm{HbA}_{1 \mathrm{c}}$ significantly decreased as BMI increased.

Table 5: Studies reporting mean levels of $\mathrm{HbA}_{1 \mathrm{c}}$ by $\mathrm{BMI}$ status.

\begin{tabular}{|c|c|c|c|c|c|}
\hline Reference & $\mathbf{N}$ & Normal & Over-weight & Obese & Notes \\
\hline $\begin{array}{l}\text { Aucott et at } \\
\text { (UK) [20] }\end{array}$ & 29,316 & $\begin{array}{l}\text { Not included in } \\
\text { study }\end{array}$ & $8.3 \%$ & $8.2 \%^{a}$ & $\begin{array}{l}\text { Individuals who were overweight had slightly higher mean } \\
\text { baseline } \mathrm{HbA}_{1 \mathrm{c}}(8.3 \%) \text { than those who were obese, where it } \\
\text { was } 8.2 \%(\mathrm{p}<0.001) \\
\text { Note: Individuals with normal weight were not included in the } \\
\text { study and most studies have compared vs normal, which was } \\
\text { not possible here }\end{array}$ \\
\hline $\begin{array}{c}\text { Ji et al } \\
\text { (China) }[11]\end{array}$ & 2052 & $7.3 \%$ & $7.3 \%$ & $7.6 \%$ & $\begin{array}{c}\text { Mean } \mathrm{HbA}_{1 \mathrm{c}} \text { was significantly different between normal BMI and } \\
\text { obese group (7.3\% vs 7.6\%; } \mathrm{p}<0.001) \text {, as were the differences } \\
\text { between the overweight and obese groups }(7.3 \% \text { vs } 7.6 \% \text {; } \\
\qquad \mathrm{p} \leq 0.002)\end{array}$ \\
\hline $\begin{array}{c}\text { Ma et al } \\
\text { (China) [22] }\end{array}$ & 17,259 & $6.7 \%$ & $7.2 \%$ & $7.7 \%$ & $\begin{array}{c}\text { Mean } \mathrm{HbA}_{1 \mathrm{c}} \text { was significantly }(\mathrm{p}<0.001) \text { worse in individuals } \\
\text { with obesity }(7.7 \%) \text { and overweight individuals }(7.2 \%) \text { vs } \\
\text { normal-weight individuals }(6.7 \%)\end{array}$ \\
\hline $\begin{array}{l}\text { Salinero-Fort } \\
\text { et al (Spain) } \\
\quad[17]\end{array}$ & 3443 & \multicolumn{3}{|c|}{$\mathrm{HbA}_{1 \mathrm{c}}$ reported by percentiles - see notes } & $\begin{array}{c}\text { Mean (and median) } \mathrm{HbA}_{1 \mathrm{c}} \text { levels significantly }(\mathrm{p}<0.001) \text { in- } \\
\text { creased with higher BMI versus the reference BMI category } \\
\text { (BMI } 23-26.8 \mathrm{~kg} / \mathrm{m}^{2} \text { ) } \\
\text { Mean } \mathrm{HbA}_{1 \mathrm{c}} \text { ranged from } 7.0 \% \text { in } \mathrm{BMI}<23 \mathrm{~kg} / \mathrm{m}^{2} \text { to } 7.3 \% \text { in BMI } \\
>39.4 \mathrm{~kg} / \mathrm{m}^{2} \\
\text { Median } \mathrm{HbA}_{1 \mathrm{c}} \text { ranged from } 6.8 \% \text { to in } \mathrm{BMI}<23 \mathrm{~kg} / \mathrm{m}^{2} \text { to } 7.1 \% \text { in } \\
\mathrm{BMI}>39.4 \mathrm{~kg} / \mathrm{m}^{2}\end{array}$ \\
\hline $\begin{array}{l}\text { Weng et al } \\
\text { (USA) [24] }\end{array}$ & 626,386 & $\begin{array}{l}\text { BMI }<30 \mathrm{~kg} / \\
\text { Note: This is a w } \\
\text { would be consic }\end{array}$ & $\begin{array}{l}\mathrm{m}^{2}=7.2 \% \\
\text { ler range than } \\
\text { ered 'normal' }\end{array}$ & $\begin{array}{c}\text { BMI } 30 \text { to }<35 \mathrm{~kg} / \mathrm{m}^{2} \\
=7.4 \% \\
\text { BMI } 35 \text { to }<40 \mathrm{~kg} / \mathrm{m}^{2} \\
=7.5 \% \\
\text { BMI } \geq 40 \mathrm{~kg} / \mathrm{m}^{2}= \\
7.5 \%\end{array}$ & $\begin{array}{l}\text { Mean } \mathrm{HbA}_{1 \mathrm{c}} \text { ranged from } 7.2 \% \text { in individuals who did not have } \\
\text { obesity to } 7.5 \% \text { in those in the two highest obesity categories } \\
\text { No statistical tests for differences were used to differentiate } \\
\text { among patients in the four BMI categories }\end{array}$ \\
\hline $\begin{array}{l}\text { Zhang et al } \\
\text { (China) [25] }\end{array}$ & 3224 & $\begin{array}{r}\text { Five d } \\
\mathrm{HbA}_{1 \mathrm{c}} \\
\text { ranging } \\
\text { of }<21 \text {. }\end{array}$ & $\begin{array}{l}\text { fferent BMI cate } \\
\text { leclined with inc } \\
\text { rom } 9.37 \% \text { in th } \\
2 \text { to } 8.69 \text { in thos } \\
>27.33 \mathrm{~kg} / \mathrm{r}\end{array}$ & $\begin{array}{l}\text { ories. Mean } \\
\text { easing BMI, } \\
\text { se with a BMI } \\
\text { with a BMI of } \\
\end{array}$ & $\begin{array}{c}\text { Mean } \mathrm{HbA}_{1 \mathrm{c}} \text { declined with increasing BMI }(\mathrm{p}<0.001) \\
\text { Paper also reports underweight categories: } \\
\text { BMI }<21.62 \mathrm{~kg} / \mathrm{m}^{2}=9.37 \% \\
\text { BMI } 21.62-23.50 \mathrm{~kg} / \mathrm{m}^{2}=9.35 \%\end{array}$ \\
\hline
\end{tabular}

a Included.

BMI: body mass index; $\mathrm{HbA}_{1 \mathrm{c}}$ : glycated hemoglobin. 


\section{Reported by glycemic control category}

\section{Mean BMI}

Three studies $[15,19,23]$ reported average BMI levels by glycemic control. One [15] reported median BMI, with the $\mathrm{HbA}_{1 \mathrm{c}}$ categories defined as controlled $(\leq 7 \%)$ and uncontrolled $(>7 \%)$, and two reported mean $\mathrm{BMI}$ across a range of $\mathrm{HbA}_{1 \mathrm{c}}$ categories: 10 in Wan et al. [23], and five in Yamakawa et al. [19].

Both studies $[19,23]$ found significant differences in mean BMI between the different $\mathrm{HbA}_{1 \mathrm{c}}$ categories, and BMI was observed to increase with $\mathrm{HbA}_{1 \mathrm{c}}$. BMI values ranged from $24.95 \mathrm{~kg} / \mathrm{m}^{2}\left(\mathrm{HbA}_{1 \mathrm{c}}\right.$ $<6 \%$ ) to $25.60 \mathrm{~kg} / \mathrm{m}^{2}\left(\mathrm{HbA}_{1 \mathrm{c}} \geq 10 \%\right)$ [23], and from $24.6 \mathrm{~kg} / \mathrm{m}^{2}$ $\left(\mathrm{HbA}_{1 \mathrm{c}}<6.5 \%\right)$ to $26.7 \mathrm{~kg} / \mathrm{m}^{2}\left(\mathrm{HbA}_{1 \mathrm{c}}>8 \%\right)$ [19].

Another study [15] reported that the difference in median BMI between the controlled $\left(24.44 \mathrm{~kg} / \mathrm{m}^{2}\right)$ and uncontrolled groups $\left(24.10 \mathrm{~kg} / \mathrm{m}^{2}\right)$ was not significant $(\mathrm{p}=0.498)$, and in both the univariate and multivariate regression analysis higher BMI was not identified as a risk factor for poor glycemic control $\left(\mathrm{HbA}_{1 \mathrm{c}}\right.$ $>7 \%$ ).

\section{Discussion}

This review was undertaken to identify studies from realworld settings that quantified the association between BMI and glycemic control. The topic was explored in various ways, including reporting glycemic control (average values, percentage achieving control, distribution across categories) by BMI and average BMI by $\mathrm{HbA}_{1 c^{\circ}}$

The datasets included in this review were often very large, and in most cases represented very broad populations. The evidence base was, however, dominated by studies from Asia (13/17), with only four studies from Europe or the USA. Most studies included in this review reported an association between BMI and glycemic control. Eight out of 13 studies found that the rates of achieving glycemic control in individuals with T2D were significantly lower with higher levels of BMI, or that the risk of not achieving glycemic control significantly increased with higher BMI. The largest study $(\mathrm{N}=626,386)$ [24] did not undertake any statistical testing to differentiate among the four BMI categories, as the analysis was intended to be exploratory. The authors did, however, report that the prevalence of poor glycemic control increased as BMI increased. With regards to statistical analysis, it is also pertinent to note that an observed statistically significant difference does not necessarily indicate a clinically significant difference.

In those studies that reported average $\mathrm{HbA}_{1 \mathrm{c}}$ levels by $\mathrm{BMI}$, it was also found that these were generally greater in individuals with higher levels of BMI in most (4/6) studies. The two studies that reported BMI by $\mathrm{HbA}_{1 \mathrm{c}}$ level found that mean BMI was significantly higher as $\mathrm{HbA}_{1 \mathrm{c}}$ increased, although in the study that just grouped individuals into controlled or uncontrolled according to $\mathrm{HbA}_{1 \mathrm{c}}(<7 \%$ defined as controlled) there was no reported difference in median BMI.
The evidence base was relatively limited, in that few recent studies specifically focused on this topic. In the wider literature there are numerous studies that report $\mathrm{HbA}_{1 \mathrm{c}}$ and $\mathrm{BMI}$ data, but we found few that reported one of these by the other. It was the case that most of the included studies reported the relationship between BMI and glycemic control as part of a wider analysis, such as the study by Ma et al. [22] on the impact of BMI on mortality. One of the few studies to focus in detail on the association between BMI and glycemic control was by Bae et al. [2].

It is important to note, as pointed out by several researchers, that the exploratory nature of the analyses included in this review that use cross-sectional data do notallow for a directexplanation of causation and leave some questions unanswerable. Nevertheless, some studies attempted to explore what is driving the association between BMI and glycemic control. Weng et al. [24] suggested two possibilities: (1) individuals with T2D and higher BMI may be more difficult to treat than those with lower BMI, as weight loss has been correlated with improved glycemic control; or (2) patients with more poorly controlled T2D may, as a result, have higher BMIs. Cai et al. [16] proposed that individuals with obesity may be more likely to fail to achieve glycemic control because they lack the self-management skills or the resources necessary for adherence (similarly, active smokers were less likely than nonsmokers to achieve glycemic control). So, patients with higher levels of BMI have a greater unwillingness or inability to make lifestyle decisions that will improve their $\mathrm{HbA}_{1 \mathrm{c}}$. There may also be clinical inertia from the physician to initiate more aggressive therapy in those with higher BMIs. Yurgin et al. [42] reported that individuals with obesity had a lower likelihood insulin initiation than that of patients without obesity (hazard ratio $0.814, \mathrm{p}=0.01$ ). The treatment characteristics of patients were not always provided by studies. This is an important consideration as, for example, patients who are treated with insulin may have good glycemic control yet experience weight gain [43].

In contrast to most of the included studies, Zhang et al. [25] reported using descriptive statistics that patients with a higher $\mathrm{BMI}$ had lower $\mathrm{HbA}_{1 \mathrm{c}}$ measurements. The study findings are inconsistent with most of the included studies; this was also one of the smallest in the review $(\mathrm{N}=3224)$. They state that the results are likely not to be causal or reverse causal. They also note individuals can be lean because of the chronic accumulation of metabolic, inflammatory, and pathological conditions caused by lifestyle behaviors such as long-term exposure to smoking, drinking, and unhealthy diets.

Overall, the studies presented in this review suggest that there is an association between higher BMI and worse glycemic control. We cannot conclude from the design of these cross-sectional studies that glycemic control is improved through reducing BMI. However, weight loss is known to directly impact insulin sensitivity and to preserve $\beta$-cell function [6]; and there is evidence from several longitudinal studies that the glycemic control of patients 
with T2D improves with weight loss. For example, a study by McAdam-Marx et al. [44] reported that participants initiating a new glucose-lowering agent who lost $\geq 3 \%$ of their body weight from baseline to 6 months were more likely to attain their $\mathrm{HbA}_{1}$ goal of $<7 \%$ (odds ratio [OR] 3.02; 95\% confidence interval [CI] $1.94-4.70)$ versus those who gained weight $(\mathrm{p}<0.001)$. Of those who lost $\geq 3 \%$ of their body weight, $64.2 \%$ reached $\mathrm{HbA}_{1 \mathrm{c}}$ targets compared with $33.1 \%$ who remained weight stable and $38.8 \%$ who gained weight $(\mathrm{p}<0.001)$. Another example comes from an analysis of the observational Look AHEAD study (N=5145) [45], which found that the magnitude of weight loss at 1 year was strongly $(p<0.0001)$ associated with improvements in glycemic control. Those who lost $5 \%$ to $<10 \%$ of their body weight had increased odds (compared with weight-stable participants) of achieving a $0.5 \%$ reduction in $\mathrm{HbA}_{1 \mathrm{c}}$ (OR 3.52; 95\% CI 2.81-4.40). Results from a literature review and meta-analysis of clinical trials of lifestyle weight-loss interventions [46] seem to suggest that a weight loss of $>5 \%$ appears necessary for beneficial effects (including to $\mathrm{HbA}_{1 \mathrm{c}}$ ), although the authors did acknowledge that achieving this level of weight loss requires intensive work and might not be a realistic primary treatment strategy for improved glycemic control.

A recent literature review [6] on the impact of weight change in adults with T2D reported mixed findings, concluding that further real-world studies were needed to advance understanding of the incremental benefits of weight loss in individuals with T2D. A key observation was that in studies included in their review, the weight-loss period evaluated was concurrent with the change in glycemic control; so the same time period was used to evaluate both the predictor and outcome variables whereas to demonstrate a causal effect between weight change and glycemic control, the weight change would have to precede the measurement of glycemic control. They outline various considerations that should be reflected in study design - such as exploring potential biases that may occur because of differences in participants' baseline characteristics, assessing the impact of varying weight-loss interventions, and determining how best to measure changes in these parameters and their relationships over time.

It should be recognized that there are benefits of reducing weight in patients who have overweight or obesity, beyond any impact on glycemic control. In those with T2D, weight loss has been shown to reduce cardiovascular risk factors and improve quality of life, mobility, and physical and sexual function [47].

Limitations of our review relate to the search itself and to the evidence base. Although the search was undertaken using a robust and reproducible protocol that retrieved $>7000$ original 'hits', it is possible that other studies relevant to the research questions were missed because it was unclear from the title or abstract that the reference held data of value. The search was restricted to English-language papers, but it is likely that other relevant studies could have been published in foreign-language journals. It also possible that further relevant studies could inevitably have been published since our searches were undertaken. The syntax used to limit studies to those undertaken in 'real-world' settings and in certain countries, and to identify studies on $\mathrm{HbA}_{1 \mathrm{c}}$ and weight, could have resulted in some relevant studies being missed. However, it should be noted that reference lists were reviewed for all included publications and no additional references were identified. As noted earlier, the geographic distribution of the included studies in this review was limited. The results from China are not generalizable to Western populations, and vice versa, with population characteristics and categories of overweight and obesity having different definitions. The prevalence of obesity in different populations also varies considerably: as shown in the review by Colosia et al. [48], prevalence rates were $6.7 \%$ in China and $64.2 \%$ in the United States. There were also differences across studies in the threshold to determine glycemic control and some used ranges rather than a cut-off in response to the call for individualized $\mathrm{HbA}_{1 \mathrm{c}}$ targets.

An advantage of many of the included studies was that the datasets were sourced from large EMR databases; but medical records cannot capture all the variables that impact glycemic control (e.g. self-monitoring of blood glucose, adherence to T2D therapy, physical activity), so these factors could not be included in the analyses. Furthermore, much of the data came from studies that addressed different research questions; many did not attempt to control for confounders (e.g. the use of different anti-diabetic medications) in exploring the specific relationship between BMI and $\mathrm{HbA}_{1 \mathrm{c}}$. Furthermore, although the focus of this study was on the link between BMI and glycemic control, it is possible that other measures warrant closer attention; for example, Chen et al. [10] found waist circumference to be an independent risk factor for poor glycemic control.

\section{Conclusion}

This review is intended as a resource that consolidates and reports the recent evidence base on glycemic control in individuals with T2D reported by BMI in real-life settings in selected countries. Most of the identified studies demonstrated that rates of achieving glycemic control in individuals with T2D were lower with higher levels of BMI, or that the risk of not achieving glycemic control increased with higher BMI. Average $\mathrm{HbA}_{1 \mathrm{c}}$ levels were generally higher in individuals with greater BMI, and individuals with higher $\mathrm{HbA}_{1 \mathrm{c}}$ tended to have higher BMI.

Given that the evidence base is dominated by studies from China and Japan (13/17 studies), there is a real need for additional studies in Europe and the United States to represent local populations, especially given the increasing prevalence of obesity and T2D. Most of the included studies had wider objectives than our specific study question, highlighting the need for large, robust, focused studies to bridge this gap. 
Nevertheless, this review consolidates data from several studies and helps to identify patients at risk of poor glycemic outcomes; in doing so, it may enable targeted healthcare strategies to reduce the burden of T2D. Focused efforts are needed particularly in individuals with T2D and obesity who, for a range of reasons, can be particularly challenging to manage. Weight management should be an integral part of the management and treatment of T2D, and the weight effects of pharmacotherapy should be considered when treatment decisions are being made.

\section{Acknowledgements}

This study was funded by Eli Lilly and Company (Indianapolis, IN, USA). The authors thank Mick Arber (York Health Economic Consortium [YHEC]) for assistance with the literature search, and Susan Robinson and Alison Terry for assistance with writing and editing, respectively.

\section{Conflict of Interest}

Kristina S Boye is an employee and minor shareholder of Eli Lilly and Company. Tessa Kennedy-Martin and Matthew KennedyMartin are employees of KMHO, who received funding from Eli Lilly for time spent conducting this research.

\section{Compliance with Ethics Guidelines}

This article is based on previously conducted studies and does not contain any new studies with human participants or animals performed by any of the authors. All named authors meet the International Committee of Medical Journal Editors (ICMJE) criteria for authorship for this article, take responsibility for the integrity of the work, and have given their approval for this version to be published.

\section{Data Availability}

Data sharing is not applicable to this article as no datasets were generated or analyzed during the current study.

\section{References}

1. Van Gaal L, Sheen A (2015) Weight management in type 2 diabetes: current and emerging approaches to treatment. Diabetes Care 38(6): 1161-1172.

2. Bae JP, Lage MJ, Mo D, Nelson DR, Hoogwerf BJ (2016) Obesity and glycemic control in patients with diabetes mellitus: Analysis of physician electronic health records in the US from 2009-2011. J Diabetes Complications 30(2): 212-220.

3. https://diabetesatlas.org/en/resources/.

4. Davies MI, D’Alessio DA, Fradkin J, Kernan WN, Mathieu C, et al (2018) Management of hyperglycemia in type 2 diabetes, 2018. A consensus report by the American Diabetes Association (ADA) and the European Association for the Study of Diabetes (EASD). Diabetes Care 41(12): 2669-2701.

5. https://www.cdc.gov/diabetes/data/statistics-report/riskscomplications.html.
6. Fridman M, Lucas ME, Paprocki Y, Dang-Tan T, Iyer NN (2020) Impact of weight change in adults with type 2 diabetes mellitus: a literature review and critical analysis. Clinicoecon Outcomes Res 12: 555-566.

7. https://care.diabetesjournals.org/highwire/filestream/59492/field_ highwire_adjunct_files/0/Standards_of_Care_2020.pdf.

8. Karkare S, Fridman M, Dang-Tan T, Lu J, Smolarz BG, et al (2019) Effect of weight change on economic outcomes among persons with type 2 diabetes mellitus in the United States: beyond glycemic control. J Manag Care Spec Pharm 25(6): 658-668.

9. https://www.cdc.gov/diabetes/library/features/diabetes-statreport.html.

10. Chen R, Ji L, Chen L, Chen L, Cai D, et al (2015) Glycemic control rate of T2DM outpatients in China: a multi-center survey. Med Sci Monit 21: 1440-1446.

11. Ji L, Zou D, Liu L, Qian L, Kadziola Z, et al (2015) Increasing body mass index identifies Chinese patients with type 2 diabetes mellitus at risk of poor outcomes. J Diabetes Complications 29(4): 488-496.

12. Liu L, Lou Q, Guo X, Yuan L, Shen L, et al. (2015) Chinese Diabetes Education Status Survey Study Group. Management status and its predictive factors in patients with type 2 diabetes in China: a nationwide multicenter study. Diabetes Metab Res Rev 31(8): 811-816.

13. Wang J, Wang MY, Wang H, Liu H-W, Lu R, et al. (2020) Status of glycosylated hemoglobin and prediction of glycemic control among patients with insulin-treated type 2 diabetes in North China: a multicenter observational study. Chin Med J (Engl) 133(1): 17-24.

14. Yokoyama H, Oishi M, Takamura H, Yamasaki K, Shirabe SI, et al. (2016) Large-scale survey of rates of achieving targets for blood glucose, blood pressure, and lipids and prevalence of complications in type 2 diabetes (JDDM 40). BMJ Open Diabetes Res Care 4(1): e000294.

15. Zhu HT, Yu M, Hu H, He QF, Pan J, et al. (2019) Factors associated with glycemic control in community-dwelling elderly individuals with type 2 diabetes mellitus in Zhejiang, China: a cross-sectional study. BMC Endocr Disord 19(1): 57

16. Cai X, Hu D, Pan C, Li G, Lu J, et al. (2019) The risk factors of glycemic control, blood pressure control, lipid control in Chinese patients with newly diagnosed type 2 diabetes: a nationwide prospective cohort study. Sci Rep 9(1): 7709.

17. Salinero-Fort MA, San Andrés-Rebollo FJ, Gómez-Campelo P, de Burgos-Lunar C, Cárdenas-Valladolid J, et al. (2017) MADIABETES Research Group. Body mass index and all-cause mortality among type 2 diabetes mellitus patients: findings from the 5 -year follow-up of the MADIABETES cohort. Eur J Intern Med 43: 46-52.

18. Tobe K, Maegawa H, Tabuchi H, Nakamura I, Uno S (2019) Impact of body mass index on the efficacy and safety of ipragliflozin in Japanese patients with type 2 diabetes mellitus: a subgroup analysis of 3-month interim results from the Specified Drug Use Results Survey of Ipragliflozin Treatment in Type 2 Diabetic Patients: Long-term Use study. J Diabetes Investig 10(5): 1262-1271.

19. Yamakawa T, Sakamoto R, Takahashi K, Suzuki J, Matuura-Shinoda M, et al. (2019) Dietary survey in Japanese patients with type 2 diabetes and the influence of dietary carbohydrate on glycated hemoglobin: the Sleep and Food Registry in Kanagawa study. J Diabetes Investig 10(2): 309-317.

20. Aucott LS, Philip S, Avenell A, Afolabi E, Sattar N, et al. (2016) Scottish Diabetes Research Network Epidemiology Group. Patterns of weight change after the diagnosis of type 2 diabetes in Scotland and their relationship with glycaemic control, mortality and cardiovascular outcomes: a retrospective cohort study. BMJ Open 6(7): e010836. 
21. Li J, Chattopadhyay K, Xu M, Chen Y, Hu F, et al. (2018) Glycaemic control in type 2 diabetes patients and its predictors: a retrospective database study at a tertiary care diabetes centre in Ningbo, China. BMJ Open 8(3): e019697.

22. Ma J, Wang X, Zheng M, Yu H, Ma J, et al. (2019) A multicenter largescale retrospective analysis of the correlation between body mass index and all-cause mortality in patients with type 2 diabetes mellitus: a seven-year real-world study. Endocr Res 44(3): 103-109.

23. Wan EYF, Yu EYT, Fung CSC, Chin WY, Fong DYT, et al. (2018) Relation between $\mathrm{HbA}_{1 \mathrm{c}}$ and incident cardiovascular disease over a period of 6 years in the Hong Kong population. Diabetes Metab 44(5): 415-423.

24. Weng W, Tian Y, Kimball ES, Kong SX, Bouchard J, et al. (2017) Treatment patterns and clinical characteristics of patients with type 2 diabetes mellitus according to body mass index: findings from an electronic medical records database. BMJ Open Diabetes Res Care 5(1) e000382.

25. Zhang Y, Guo Y, Shen X, Zhao F, Yan S (2019) Lower body mass index is not of more benefit for diabetic complications. J Diabetes Investig 10(5): 1307-1317.

26. American Diabetes Association (2017) Standards of medical care in diabetes. Diabetes Care 40(suppl 1): S11-S74.

27. Weng J, Ji L, Jia W, Lu J, Zhou Z, et al. (2016) Chinese Diabetes Society. Standards of care for type 2 diabetes in China. Diabetes Metab Res Rev 32(5): 442-458.

28. Zhou B (2002) Cooperative Meta-Analysis Group of China Obesity Task Force Predictive values of body mass index and waist circumference to risk factors of related diseases in Chinese adult population. Chinese J Epidemiol 23: 5-10.

29. http:// iris.wpro.who.int/ bitstream/ handle/10665.1/ 11408/9789290617280_eng.pdf; jsessionid = 9D2E0398D69E2CD263CF56D229F50EC0? sequence $=1$.

30. Chinese Diabetes Society (2013) China guideline for type 2 diabetes. Chin J Endocrinol Metab 30: 893-942.

31. American Diabetes Association (2015) Standards of medical care in diabetes. Diabetes Care 38(suppl 2): S1-S93.

32. Diabetes Society of the Chinese Medical Association (2017) Guidelines for the prevention and treatment of type 2 diabetes in China. Chin J Diabetes Mellit 1: 4-67.

33. Yang W, Han P, Min KW, Wang B, Mansfield T, et al. (2016) Efficacy and safety of dapagliflozin in Asian patients with type 2 diabetes after metformin failure: a randomized controlled trial. J Diabetes 8: 796808

34. Japanese Society for the Study of Obesity Diseases (2016) Guidelines for the Management of Obesity Disease. Tokyo: Asakura-shoten.
35. Haneda M, Noda M, Origasa H, Noto H, Yabe D, et al. (2018) Japanese Clinical Practice Guideline for Diabetes 2016. Diabetol Int 9(1): 1-45.

36. American Diabetes Association (2011) Standards of medical care in diabetes. Diabetes Care 34(suppl 1): S11-S61.

37. Chiu CJ, Wray LA, Lu FH, Beverly EA (2013) BMI change patterns and disability development of middle-aged adults with diabetes: a dual trajectory modeling approach. J Gen Intern Med 28(9): 1150-1156.

38. Berkowitz SA, Krumme AA, Avorn J, Brennan T, Matlin OS, et al. (2014) Initial choice of oral glucose-lowering medication for diabetes mellitus: a patient-centered comparative effectiveness study. JAMA Intern Med 174(12): 1955-1962.

39. http://apps.who.int/bmi/index.jsp?introPage=intro_3.html.

40. Yau CK, Eng C, Cenzer IS, Boscardin WJ, Rice-Trumble K, et al. (2012) Glycosylated hemoglobin and functional decline in communitydwelling nursing home - eligible elderly adults with diabetes mellitus. J Am Geriatr Soc 60(7): 1215-1221.

41. Nicholas J, Charlton J, Dregan A, Gulliford MC (2013) Recent HbA values and mortality risk in type 2 diabetes. Population-based casecontrol study. PLoS One 8(7): e68008.

42. Yurgin N, Secnik K, Lage MJ (2008) Obesity and the use of insulin: a study of patients with type 2 diabetes in the UK. J Diabetes Complications 22(4): 235-240.

43. Brown A, Guess N, Dornhorst A, Taheri S, Frost G (2017) Insulinassociated weight gain in obese type 2 diabetes mellitus patients: What can be done? Diabetes Obes Metab 19(12): 1655-1668.

44. McAdam-Marx C, Bellows BK, Unni S, Mukherjee J, Wygant G, et al. (2014) Determinants of glycaemic control in a practice setting: the role of weight loss and treatment adherence (The DELTA Study). Int J Clin Pract 68(11): 1309-1317.

45. Wing RR, Lang W, Wadden TA, Safford M, Knowler WC, et al. (2011) Look AHEAD Research Group. Benefits of modest weight loss in improving cardiovascular risk factors in overweight and obese individuals with type 2 diabetes. Diabetes Care 34(7): 1481-1486.

46. Franz MJ, Boucher JL, Rutten-Ramos S, VanWormer JJ (2015) Lifestyle weight-loss intervention outcomes in overweight and obese adults with type 2 diabetes: a systematic review and meta-analysis of randomized clinical trials. J Acad Nutr Diet 115(9): 1447-1463.

47. Wilding JPH (2014) The importance of weight management in type 2 diabetes mellitus. Int J Clin Pract 68(6): 682-691.

48. Colosia AD, Palencia R, Khan S (2013) Prevalence of hypertension and obesity in patients with type 2 diabetes mellitus in observational studies: a systematic literature review. Diabetes Metab Syndr Obes 6: 327-338. 
This work is licensed under Creative Commons Attribution 4.0 Licen

DOI: 10.19080/CRDOJ.2021.15.555904

Your next submission with Juniper Publishers
will reach you the below assets
- Quality Editorial service
- Swift Peer Review
- Reprints availability
- E-prints Service
- Manuscript Podcast for convenient understanding
- Global attainment for your research
- Manuscript accessibility in different formats
( Pdf, E-pub, Full Text, Audio)
- Unceasing customer service
Track the below URL for one-step submission
https://juniperpublishers.com/online-submission.php

